\title{
SCHATTEN CLASSES OF INTEGRATION OPERATORS ON DIRICHLET SPACES
}

\author{
JORDI PAU AND JOSÉ ÁNGEL PELÁEZ
}

\begin{abstract}
We address the question of describing the membership to Schatten-Von Neumann ideals $\mathcal{S}_{p}$ of integration operators $\left(T_{g} f\right)(z)=$ $\int_{0}^{z} f(\zeta) g^{\prime}(\zeta) d \zeta$ acting on Dirichlet type spaces. We also study this problem for multiplication, Hankel and Toeplitz operators. In particular, we provide an extension of Luecking's result on Toeplitz operators [10, p. $347]$.
\end{abstract}

\section{IntRoduCtion AND MAIN RESUlts}

Let $\mathbb{D}$ denote the open unit disk of the complex plane, and let $H(\mathbb{D})$ be the class of all analytic functions on $\mathbb{D}$. For $\alpha>-1$, let

$$
d A_{\alpha}(z)=(\alpha+1)\left(1-|z|^{2}\right)^{\alpha} d A(z),
$$

where $d A(z)=\frac{1}{\pi} d x d y$ is the normalized area measure on $\mathbb{D}$. For $\alpha \geq 0$, the weighted Dirichlet-type space $\mathcal{D}_{\alpha}$ consists of those functions $f \in H(\mathbb{D})$ for which

$$
\|f\|_{\mathcal{D}_{\alpha}}^{2} \stackrel{\text { def }}{=}|f(0)|^{2}+\int_{\mathbb{D}}\left|f^{\prime}(z)\right|^{2} d A_{\alpha}(z)<\infty
$$

Note that the space $\mathcal{D}_{0}$ is just the classical Dirichlet space and, as usual, will be simply denoted by $\mathcal{D}$. The spaces $\mathcal{D}_{\alpha}$ are reproducing kernel Hilbert spaces: for each $z \in \mathbb{D}$, there are functions $K_{z}^{\alpha} \in \mathcal{D}_{\alpha}$ for which the reproducing formula $f(z)=\left\langle f, K_{z}^{\alpha}\right\rangle_{\mathcal{D}_{\alpha}}$ holds, where the inner product in $\mathcal{D}_{\alpha}$ is given by

$$
\langle f, g\rangle_{\mathcal{D}_{\alpha}} \stackrel{\text { def }}{=} f(0) \overline{g(0)}+\int_{\mathbb{D}} f^{\prime}(z) \overline{g^{\prime}(z)} d A_{\alpha}(z) .
$$

For $0<p<\infty$, we shall also write $A_{\alpha}^{p}$ for the weighted Bergman space of those $g \in H(\mathbb{D})$ such that

$$
\|g\|_{A_{\alpha}^{p}}^{p}=\int_{\mathbb{D}}|g(z)|^{p} d A_{\alpha}(z)<\infty .
$$

Date: April 16, 2012.

Key words and phrases. Dirichlet spaces, Schatten classes, integration operators, Toeplitz operators.

The first author is supported by SGR grant 2009SGR 420 (Generalitat de Catalunya) and DGICYT grant MTM2011-27932-C02-01 (MCyT/MEC), while the second author is supported by: the Ramón y Cajal program of MICINN (Spain), "the Ministerio de Educación y Ciencia, Spain" (MTM2011-25502) and from "La Junta de Andalucía" (FQM210), (P09-FQM-4468). 
Here we put our attention on the study of the integration operator $T_{g}$ and the multiplication operator $M_{g}$ defined by

$$
\begin{aligned}
& \left(T_{g} f\right)(z)=\int_{0}^{z} f(\zeta) g^{\prime}(\zeta) d \zeta, \\
& M_{g}(f)=g(z) f(z) .
\end{aligned}
$$

where $g$ is an analytic function on $\mathbb{D}$. The bilinear operator $(f, g) \rightarrow \int f g^{\prime}$ was introduced by A. Calderón in harmonic analysis in the 60's for his research on commutators of singular integral operators [8] (see also [25, p.1136]). After that, it and different variations going by the name of "paraproducts", have been extensively studied, becoming fundamental tools in harmonic analysis. Pommerenke was probably one of the first authors of the complex function theory community to consider the operator $T_{g}$ [17]. After the pioneering works of Aleman and Siskakis $[4,5]$, the study of the operator $T_{g}$ on several spaces of analytic functions has attracted a lot of attention in recent years (see $[2,3,14,16,22,23]$ ).

Our main goal is to study the membership in the Schatten-Von Neumann ideals $\mathcal{S}_{p}$ of the integration operator $T_{g}: \mathcal{D}_{\alpha} \rightarrow \mathcal{D}_{\alpha}$. If $\alpha>1, \mathcal{D}_{\alpha}$ is nothing else but $A_{\alpha-2}^{2}$ and $\mathcal{D}_{1}=H^{2}$, the classical Hardy space, so for $p>1$, then $T_{g} \in \mathcal{S}_{p}\left(\mathcal{D}_{\alpha}\right)$ if and only if $g$ belongs to the Besov space $B_{p}$, and if $0<p \leq 1$, then $T_{g} \in \mathcal{S}_{p}\left(\mathcal{D}_{\alpha}\right)$ if and only if $g$ is constant (see $[4,5]$ ). We recall that, for $p>1$, the Besov space $B_{p}$ is the space of all analytic functions $g$ in $\mathbb{D}$ such that

$$
\int_{\mathbb{D}}\left|g^{\prime}(z)\right|^{p}\left(1-|z|^{2}\right)^{p} d \lambda(z)<\infty,
$$

where $d \lambda(z)=\frac{d A(z)}{\left(1-|z|^{2}\right)^{2}}$ is the hyperbolic measure on $\mathbb{D}$.

The following result is implicit in the literature (see [27]) and can be proved by using the theory of Toeplitz operators (see Section 5).

Theorem A. Let $g \in H(\mathbb{D})$. We have the following:

(a) Let $0<\alpha<1$ and $p>1$ with $p(1-\alpha)<2$. Then $T_{g} \in S_{p}\left(\mathcal{D}_{\alpha}\right)$ if and only if $g$ belongs to $B_{p}$.

(b) If $0<p \leq 1$ and $0<\alpha<1$, then $T_{g} \in S_{p}\left(\mathcal{D}_{\alpha}\right)$ if and only if $g$ is constant.

However for $0<\alpha<1$ and $p(1-\alpha) \geq 2$, to the best of our knowledge, it is an open problem founding a description of those $g \in H(\mathbb{D})$ such that $T_{g} \in S_{p}\left(\mathcal{D}_{\alpha}\right)$. This motivation leads us to introduce for $0 \leq \alpha<\infty$ and $1<p<\infty$, the space $X_{\alpha}^{p}$ which consists of those $g \in H(\mathbb{D})$ such that

$$
\|g\|_{X_{\alpha}^{p}}^{p} \stackrel{\text { def }}{=}|g(0)|^{p}+\int_{\mathbb{D}}\left(\left(1-|w|^{2}\right)^{\alpha} \int_{\mathbb{D}} \frac{\left|g^{\prime}(z)\right|^{2} d A_{\alpha}(z)}{|1-\bar{w} z|^{2+2 \alpha}}\right)^{p / 2}\left(1-|w|^{2}\right)^{p-2} d A(w)<\infty .
$$

The following result gives a description of the membership in $\mathcal{S}_{p}\left(\mathcal{D}_{\alpha}\right)$ in the range $p>1$ and $p(1-\alpha)<4$. 
Theorem 1. Let $0<\alpha<1, g \in H(\mathbb{D})$ and $p>1$ with $p(1-\alpha)<4$. Then $T_{g} \in S_{p}\left(\mathcal{D}_{\alpha}\right)$ if and only if $g$ belongs to $X_{\alpha}^{p}$.

Now we are going to deal with the case of the classical Dirichlet space $\mathcal{D}$. The situation here it seems to be more difficult. First of all, it is easy (and well known) to describe when the operator $T_{g}$ belongs to the HilbertSchmidt class $\mathcal{S}_{2}(\mathcal{D})$. Indeed, for any orthonormal basis $\left\{e_{n}\right\}$ of the Dirichlet space, one has (see Section 2)

$$
\begin{aligned}
\left\|T_{g}\right\|_{\mathcal{S}_{2}}^{2}=\sum_{n}\left\|T_{g} e_{n}\right\|_{\mathcal{D}}^{2} & =\int_{\mathbb{D}}\left|g^{\prime}(z)\right|^{2} \sum_{n}\left|e_{n}(z)\right|^{2} d A(z) \\
& =\int_{\mathbb{D}}\left|g^{\prime}(z)\right|^{2} \log \frac{e}{1-|z|^{2}} d A(z) .
\end{aligned}
$$

Therefore, the integration operator $T_{g}$ belongs to $\mathcal{S}_{2}(\mathcal{D})$ if and only if the last integral in the previous equation is finite. The class of functions $g \in H(\mathbb{D})$ satisfying this condition shall be denoted by $\mathcal{D} \mathcal{L}$.

If $1<p<2$ Theorem A suggests that the membership in $\mathcal{S}_{p}(\mathcal{D})$ of the operator $T_{g}$ could be described by those $g$ being in the Besov space $B_{p}$. However, since for $p<2$ any operator on $\mathcal{S}_{p}$ must be Hilbert-Schmidt, clearly the condition $g \in \mathcal{D} \mathcal{L}$ is necessary for $T_{g}$ being in $\mathcal{S}_{p}(\mathcal{D})$, and an easy calculation shows that the function $g(z)=\log \log \frac{e}{1-z}$ belongs to $B_{p}$ for all $p>1$ but $g$ is not in $\mathcal{D} \mathcal{L}$. Thus, the condition $g \in B_{p}$ is not sufficient to assert that $T_{g}$ is in $\mathcal{S}_{p}(\mathcal{D})$.

On the other hand, as in the weighted case, there are no trace class integration operators in the Dirichlet space unless $g$ is constant.

TheOREM 2. Let $0<p \leq 1$ and $g \in H(\mathbb{D})$. Then $T_{g} \in \mathcal{S}_{p}(\mathcal{D})$ if and only if $g$ is constant.

For the case $1<p<2$ we have a necessary condition and a different sufficient condition. We will see that they are sharp in a certain sense. Before that, for $p>1$ and $\gamma>0$, we consider the space $B_{p, \log ^{\gamma} \text {, that }}$ consists of those functions $g$ analytic on $\mathbb{D}$ such that

$$
\|g\|_{B_{p, \log \gamma}}^{p}=|g(0)|^{p}+\int_{\mathbb{D}}\left|g^{\prime}(z)\right|^{p}\left(\log \frac{e}{1-|z|}\right)^{\gamma}\left(1-|z|^{2}\right)^{p-2} d A(z)<\infty .
$$

TheOREM 3. Let $1<p<2$ and $g \in H(\mathbb{D})$. Then

(a) If $T_{g} \in \mathcal{S}_{p}(\mathcal{D})$, then $g \in B_{p}$.

(b) If $g \in B_{p, \log ^{p / 2}}$, then $T_{g} \in \mathcal{S}_{p}(\mathcal{D})$. Moreover, $\left\|T_{g}\right\|_{\mathcal{S}_{p}} \leq C\|g\|_{B_{p, \log p / 2}}$.

(c) If $g \in X_{0}^{p}$, then $T_{g} \in \mathcal{S}_{p}(\mathcal{D})$.

When one takes the monomials as the symbols, it turns out that the correct behavior of $\left\|T_{g}\right\|_{\mathcal{S}_{p}}$ is given by $B_{p}$ or $X_{0}^{p}$, while if one takes as a symbol to be functions of the type $g_{a}(z)=(1-\bar{a} z)^{-\gamma}$, the correct behavior is given by the $B_{p, \log ^{p / 2}}$ condition (see Lemmas 4.1 and 4.2 ). 
The case $p>2$ seems to be a mystery. Let $\mathcal{D}_{\beta}^{p}$ denote the space of those functions $f$ with $f^{\prime} \in A_{\beta}^{p}$. For $p>2$, the inclusion $\mathcal{D}_{\beta}^{p} \subset \mathcal{D}$ holds if and only if $\beta<(p-2) / 2$; and $\mathcal{D} \subset \mathcal{D}_{\beta}^{p}$ if and only if $\beta \geq p-2$ (see [28, p.94]). Thus, if one is looking for conditions on the integrability of $g^{\prime}$, it can not be expected some necessary condition much better than $B_{p}=\mathcal{D}_{p-2}^{p}$, and a sufficient condition must be stronger than $g$ being in $\mathcal{D}_{\frac{p-2}{2}}^{p}$. We will discuss a little bit this case in Section 4 .

We close this section saying that from now on the paper is organized as follows. In Section 2 we introduce several preliminary general results related on Schatten classes of operators on Dirichlet spaces. Section 3 is devoted to the proof of Theorem 1. There it will be proved directly (see Proposition 3.1 (iv)) the identity

$$
X_{\alpha}^{p}=B_{p}, \quad p>1, \alpha>0, \text { and } p(1-\alpha)<2,
$$

which together with Theorem 1 gives a proof of Theorem A not relying in the theory of Toeplitz operators. It is worth mentioning that the Besov space $B_{p}$ is rich of several characterizations (the identity (1.3) gives a new one), each of them being the appropriate tool to use in different situations (see [1], [7], or [29] for example). In Section 4 we prove Theorem 2 and Theorem 3. Also, by using some testing classes of functions, we show that those results are sharp in a certain sense. Finally, Section 5 is devoted to study the relationship of the integration operator $T_{g}$ with other classical operators acting on weighted Dirichlet spaces, such as Toeplitz operators, multiplication operators or big and small Hankel operators. A similar connection also happens in other contexts [18]. Indeed, the same techniques used in the proof of Theorem 1 work to demonstrate an extension for positive Borel measures of the helpful result of Luecking on Toeplitz operators [10, p. 347]).

Throughout the paper, the letter $C$ will denote a positive absolute constant whose value may change at different occurrences, and we write $A \asymp B$ when the two quantities $A$ and $B$ are comparable.

\section{Preliminary Results}

Let $H$ and $K$ be separable Hilbert spaces. Given $0<p<\infty$, let $\mathcal{S}_{p}(H, K)$ denote the Schatten $p$-class of operators from $H$ to $K$. If $H=K$ we simply shall write $\mathcal{S}_{p}(H)$. The class $\mathcal{S}_{p}(H, K)$ consists of those compact operators $T$ from $H$ to $K$ with its sequence of singular numbers $\lambda_{n}$ belonging to $\ell^{p}$, the $p$-summable sequence space. We recall that the singular numbers of a compact operator $T$ are the square root of the eigenvalues of the positive operator $T^{*} T$, where $T^{*}$ denotes the Hilbert adjoint of $T$. We remind the reader that $T \in \mathcal{S}_{p}(H)$ if and only if $T^{*} T \in \mathcal{S}_{p / 2}(H)$. Also, the compact 
operator $T$ admits a decomposition of the form

$$
T=\sum_{n} \lambda_{n}\left\langle\cdot, e_{n}\right\rangle_{H} \sigma_{n}
$$

where $\left\{\lambda_{n}\right\}$ are the singular numbers of $T,\left\{e_{n}\right\}$ is an orthonormal set in $H$, and $\left\{\sigma_{n}\right\}$ is an orthonormal set in $K$.

For $p \geq 1$, the class $\mathcal{S}_{p}(H, K)$ is a Banach space equipped with the norm

$$
\|T\|_{\mathcal{S}_{p}}=\left(\sum_{n}\left|\lambda_{n}\right|^{p}\right)^{1 / p},
$$

while for $0<p<1$ one has the inequality $\|S+T\|_{\mathcal{S}_{p}}^{p} \leq\|S\|_{\mathcal{S}_{p}}^{p}+\|T\|_{\mathcal{S}_{p}}^{p}$. We refer to [21] or [30, Chapter 1] for a brief account on the theory of Schatten $p$-classes.

We shall write $H$ for a Hilbert space of analytic functions in $\mathbb{D}$ with reproducing kernels $K_{z}$. Given an operator $T$ on $H$, usually the reproducing kernel functions carry a large amount of information about relevant properties of $T$, such as boundedness, compactness, membership in Schatten $p$-classes, etc. It is known that if $\left\{e_{n}\right\}$ is an orthonormal basis of a Hilbert space $H$ of analytic functions in $\mathbb{D}$ with reproducing kernel $K_{z}$, then

$$
K_{z}(\zeta)=\sum_{n} e_{n}(\zeta) \overline{e_{n}(z)}
$$

for all $z$ and $\zeta$ in $\mathbb{D}$, see e.g. [30, Theorem 4.19]. We also introduce $J_{z}$, the derivative of $K_{z}$ respect to $\bar{z}$, that is,

$$
J_{z}(\zeta)=\sum_{n} e_{n}(\zeta) \overline{e_{n}^{\prime}(z)}
$$

It follows that

$$
\sum_{n}\left|e_{n}(z)\right|^{2} \leq\left\|K_{z}\right\|_{H}^{2}, \quad \text { and } \quad \sum_{n}\left|e_{n}^{\prime}(z)\right|^{2} \leq\left\|J_{z}\right\|_{H}^{2}
$$

for any orthonormal set $\left\{e_{n}\right\}$ of $H$, and equality in (2.3) holds if $\left\{e_{n}\right\}$ is an orthonormal basis of $H$. We shall write $k_{z}$ and $j_{z}$ for the normalizations of these functions.

In order to avoid some confusions when dealing with reproducing kernels of either $\mathcal{D}_{\alpha}$ or $A_{\alpha}^{2}$, we use the notation $B_{z}^{\alpha}$ for the reproducing kernel of the weighted Bergman space $A_{\alpha}^{2}$ at the point $z$, and let $b_{z}^{\alpha}=\frac{B_{z}^{\alpha}}{\left\|B_{z}^{\alpha}\right\|_{A_{\alpha}^{2}}}$ be its normalization. It is well known (see [30, Corollary 4.20]) that

$$
B_{z}^{\alpha}(w)=\frac{1}{(1-\bar{z} w)^{2+\alpha}}, \quad \text { and } \quad\left\|B_{z}^{\alpha}\right\|_{A_{\alpha}^{2}}=\left(1-|z|^{2}\right)^{-\frac{(2+\alpha)}{2}} .
$$

The reproducing kernel function for the Dirichlet type space $\mathcal{D}_{\alpha}$ is denoted by $K_{z}^{\alpha}$, and $k_{z}^{\alpha}$ denotes the corresponding normalized reproducing kernel. Since $f \in \mathcal{D}_{\alpha}$ if and only if $f^{\prime} \in A_{\alpha}^{2}$, using the reproducing formula for 
the Bergman space $A_{\alpha}^{2}$ (see [30, Proposition 4.23]), it can be deduced the following expression of the reproducing kernel of $\mathcal{D}_{\alpha}$ (see [7] or [27]):

$$
K_{z}^{\alpha}(w)=1+\int_{0}^{w} \int_{0}^{\bar{z}} \frac{d \zeta}{(1-\eta \zeta)^{2+\alpha}} d \eta
$$

In particular, for $\alpha=0$,

$$
K_{z}^{\mathcal{D}}(w):=K_{z}^{0}(w)=1+\log \frac{1}{1-\bar{z} w} .
$$

Also, it is easy to see that

$$
\left\|K_{z}^{\alpha}\right\|_{\mathcal{D}_{\alpha}}^{2}=K_{z}^{\alpha}(z) \asymp\left\{\begin{array}{ccc}
\log \frac{e}{1-|z|^{2}} & \text { if } & \alpha=0 \\
\left(1-|z|^{2}\right)^{-\alpha} & \text { if } & \alpha>0
\end{array} .\right.
$$

The next two results are certainly well known to the experts (see [9] or [24] for similar results), but we find convenient for the reader to give a proof here.

Proposition 2.1. Let $T: A_{\alpha}^{2} \rightarrow H$ be a compact operator, where $H$ is any separable Hilbert space.

(i) For $p \geq 2$,

$$
\int_{\mathbb{D}}\left\|T b_{z}^{\alpha}\right\|_{H}^{p} d \lambda(z) \leq \frac{1}{1+\alpha}\|T\|_{\mathcal{S}_{p}}^{p}
$$

(ii) For $0<p \leq 2$,

$$
\|T\|_{\mathcal{S}_{p}}^{p} \leq(1+\alpha) \int_{\mathbb{D}}\left\|T b_{z}^{\alpha}\right\|_{H}^{p} d \lambda(z)
$$

Proof. Since the operator $T$ is compact, it admits the decomposition

$$
T f=\sum_{n} \lambda_{n}\left\langle f, e_{n}\right\rangle_{A_{\alpha}^{2}} f_{n}
$$

where $\left\{\lambda_{n}\right\}$ are the singular values of $T,\left\{e_{n}\right\}$ is an orthonormal set in $A_{\alpha}^{2}$, and $\left\{f_{n}\right\}$ is an orthonormal set in $H$. Then

$$
T B_{z}^{\alpha}=\sum_{n} \lambda_{n} \overline{e_{n}(z)} f_{n}
$$

and therefore

$$
\left\|T B_{z}^{\alpha}\right\|_{H}^{2}=\sum_{n}\left|\lambda_{n}\right|^{2}\left|e_{n}(z)\right|^{2}
$$


Now, if $p \geq 2$, using Hölder's inequality, (2.3) and (2.4), yields

$$
\begin{aligned}
\int_{\mathbb{D}}\left\|T b_{z}^{\alpha}\right\|_{H}^{p} d \lambda(z) & =\int_{\mathbb{D}}\left\|T B_{z}^{\alpha}\right\|_{H}^{p}\left\|B_{z}^{\alpha}\right\|_{A_{\alpha}^{2}}^{-p} d \lambda(z) \\
& =\int_{\mathbb{D}}\left(\sum_{n}\left|\lambda_{n}\right|^{2}\left|e_{n}(z)\right|^{2}\right)^{p / 2}\left\|B_{z}^{\alpha}\right\|_{A_{\alpha}^{2}}^{-p} d \lambda(z) \\
& \leq \int_{\mathbb{D}}\left(\sum_{n}\left|\lambda_{n}\right|^{p}\left|e_{n}(z)\right|^{2}\right)\left(\sum_{n}\left|e_{n}(z)\right|^{2}\right)^{\frac{p-2}{2}}\left\|B_{z}^{\alpha}\right\|_{A_{\alpha}^{2}}^{-p} d \lambda(z) \\
& \leq \sum_{n}\left|\lambda_{n}\right|^{p} \int_{\mathbb{D}}\left|e_{n}(z)\right|^{2}\left\|B_{z}^{\alpha}\right\|_{A_{\alpha}^{2}}^{-2} d \lambda(z) \\
& =\sum_{n}\left|\lambda_{n}\right|^{p} \int_{\mathbb{D}}\left|e_{n}(z)\right|^{2}\left(1-|z|^{2}\right)^{\alpha} d A(z)=\frac{1}{1+\alpha}\|T\|_{S_{p}}^{p} .
\end{aligned}
$$

If $0<p \leq 2$, a similar argument, using Hölder's inequality with exponent $2 / p \geq 1,(2.3)$ and $(2.4)$, gives

$$
\begin{aligned}
\|T\|_{S_{p}}^{p} & =(1+\alpha) \int_{\mathbb{D}} \sum_{n}\left|\lambda_{n}\right|^{p}\left|e_{n}(z)\right|^{2}\left\|B_{z}^{\alpha}\right\|_{A_{\alpha}^{2}}^{-2} d \lambda(z) \\
& \leq(1+\alpha) \int_{\mathbb{D}}\left(\sum_{n}\left|\lambda_{n}\right|^{2}\left|e_{n}(z)\right|^{2}\right)^{\frac{p}{2}}\left(\sum_{n}\left|e_{n}(z)\right|^{2}\right)^{\frac{2-p}{2}}\left\|B_{z}^{\alpha}\right\|_{A_{\alpha}^{2}}^{-2} d \lambda(z) \\
& \leq(1+\alpha) \int_{\mathbb{D}}\left(\sum_{n}\left|\lambda_{n}\right|^{2}\left|e_{n}(z)\right|^{2}\right)^{\frac{p}{2}}\left\|B_{z}^{\alpha}\right\|_{A_{\alpha}^{2}}^{-p} d \lambda(z) \\
& =(1+\alpha) \int_{\mathbb{D}}\left\|T b_{z}^{\alpha}\right\|_{H}^{p} d \lambda(z) .
\end{aligned}
$$

The corresponding analogue of Proposition 2.1 for the Dirichlet type spaces $\mathcal{D}_{\alpha}$ uses the functions $j_{z}^{\alpha} \stackrel{\text { def }}{=} \frac{J_{z}^{\alpha}}{\left\|J_{z}^{\alpha}\right\|_{\mathcal{D}_{\alpha}}}$.

Proposition 2.2. Let $T: \mathcal{D}_{\alpha} \rightarrow H$ be a compact operator, where $H$ is any separable Hilbert space.

(i) For $p \geq 2$,

$$
\int_{\mathbb{D}}\left\|T j_{z}^{\alpha}\right\|_{H}^{p} d \lambda(z) \leq \frac{1}{1+\alpha}\|T\|_{\mathcal{S}_{p}}^{p}
$$

(ii) For $0<p \leq 2$,

$$
\|T\|_{\mathcal{S}_{p}}^{p} \leq\|T\|^{p}+(1+\alpha) \int_{\mathbb{D}}\left\|T j_{z}^{\alpha}\right\|_{H}^{p} d \lambda(z) .
$$

Proof. Since $T$ is compact, it admits the decomposition

$$
T f=\sum_{n} \lambda_{n}\left\langle f, e_{n}\right\rangle_{\mathcal{D}_{\alpha}} f_{n}
$$


where $\left\{\lambda_{n}\right\}$ are the singular values of $T,\left\{e_{n}\right\}$ is an orthonormal set in $\mathcal{D}_{\alpha}$, and $\left\{f_{n}\right\}$ is an orthonormal set in $H$. It follows from $(2.5)$ that $J_{z}^{\alpha}(0)=0$, then using (2.4),

$$
\left\|J_{z}^{\alpha}\right\|_{\mathcal{D}_{\alpha}}=\left\|B_{z}^{\alpha}\right\|_{A_{\alpha}^{2}}=\left(1-|z|^{2}\right)^{-\frac{(2+\alpha)}{2}}
$$

and

$$
\left\langle J_{z}^{\alpha}, e_{n}\right\rangle_{\mathcal{D}_{\alpha}}=\left\langle B_{z}^{\alpha}, e_{n}^{\prime}\right\rangle_{A_{\alpha}^{2}}=\overline{e_{n}^{\prime}(z)} .
$$

Thus $T J_{z}^{\alpha}=\sum_{n} \lambda_{n} \overline{e_{n}^{\prime}(z)} f_{n}$, and therefore

$$
\left\|T J_{z}^{\alpha}\right\|_{H}^{2}=\sum_{n}\left|\lambda_{n}\right|^{2}\left|e_{n}^{\prime}(z)\right|^{2} .
$$

If $p \geq 2$, using the identity (2.8), Hölder's inequality, (2.3) and (2.7)

$$
\begin{aligned}
\int_{\mathbb{D}}\left\|T j_{z}^{\alpha}\right\|_{H}^{p} d \lambda(z) & =\int_{\mathbb{D}}\left(\sum_{n}\left|\lambda_{n}\right|^{2}\left|e_{n}^{\prime}(z)\right|^{2}\right)^{p / 2}\left\|J_{z}^{\alpha}\right\|_{\mathcal{D}_{\alpha}}^{-p} d \lambda(z) \\
& \leq \int_{\mathbb{D}}\left(\sum_{n}\left|\lambda_{n}\right|^{p}\left|e_{n}^{\prime}(z)\right|^{2}\right)\left(\sum_{n}\left|e_{n}^{\prime}(z)\right|^{2}\right)^{\frac{p-2}{2}}\left\|J_{z}^{\alpha}\right\|_{\mathcal{D}_{\alpha}}^{-p} d \lambda(z) \\
& \leq \sum_{n}\left|\lambda_{n}\right|^{p} \int_{\mathbb{D}}\left|e_{n}^{\prime}(z)\right|^{2}\left\|J_{z}^{\alpha}\right\|_{\mathcal{D}_{\alpha}}^{-2} d \lambda(z) \\
& =\sum_{n}\left|\lambda_{n}\right|^{p} \int_{\mathbb{D}}\left|e_{n}^{\prime}(z)\right|^{2}\left(1-|z|^{2}\right)^{\alpha} d A(z) \leq \frac{1}{1+\alpha}\|T\|_{\mathcal{S}_{p}}^{p} .
\end{aligned}
$$

If $0<p \leq 2$, since $\left\|e_{n}\right\|_{\mathcal{D}_{\alpha}}=1$, and $d A_{\alpha}(z)=(1+\alpha)\left\|J_{z}^{\alpha}\right\|_{\mathcal{D}_{\alpha}}^{-2} d \lambda(z)$ due to (2.7), then

$$
\begin{aligned}
\|T\|_{\mathcal{S}_{p}}^{p} & =\sum_{n}\left|\lambda_{n}\right|^{p}\left|e_{n}(0)\right|^{2}+(1+\alpha) \sum_{n}\left|\lambda_{n}\right|^{p} \int_{\mathbb{D}}\left|e_{n}^{\prime}(z)\right|^{2}\left\|J_{z}^{\alpha}\right\|_{\mathcal{D}_{\alpha}}^{-2} d \lambda(z) \\
& =(I)+(I I) .
\end{aligned}
$$

For the first term (I), observe that $\left|\lambda_{n}\right| \leq\|T\|$, and therefore

$$
(I) \leq\|T\|^{p} \sum_{n}\left|e_{n}(0)\right|^{2}=\|T\|^{p}\left\|K_{0}^{\alpha}\right\|_{\mathcal{D}_{\alpha}}^{2}=\|T\|^{p} .
$$

For the second term (II), due to Hölder's inequality, (2.3) and the identity $(2.8)$

$$
\begin{aligned}
(I I) & \leq(1+\alpha) \int_{\mathbb{D}}\left(\sum_{n}\left|\lambda_{n}\right|^{2}\left|e_{n}^{\prime}(z)\right|^{2}\right)^{p / 2}\left(\sum_{n}\left|e_{n}^{\prime}(z)\right|^{2}\right)^{\frac{2-p}{2}}\left\|J_{z}^{\alpha}\right\|_{\mathcal{D}_{\alpha}}^{-2} d \lambda(z) \\
& \leq(1+\alpha) \int_{\mathbb{D}}\left(\sum_{n}\left|\lambda_{n}\right|^{2}\left|e_{n}^{\prime}(z)\right|^{2}\right)^{p / 2}\left\|J_{z}^{\alpha}\right\|_{\mathcal{D}_{\alpha}}^{-p} d \lambda(z) \\
& =(1+\alpha) \int_{\mathbb{D}}\left\|T j_{z}^{\alpha}\right\|_{H}^{p} d \lambda(z) .
\end{aligned}
$$


Putting the estimates obtained for (I) and (II) in (2.9) we obtain part (ii). This completes the proof.

The following result will also be needed.

Lemma 2.3. Let $\alpha \geq 0$. If $1 \leq p<2$ there is a constant $C=C(p, \alpha)>0$ such that

$$
\sum_{n}\left|e_{n}(z)\right|^{p}\left|e_{n}^{\prime}(z)\right|^{2-p} \geq C\left(1-|z|^{2}\right)^{p-2-\alpha}, \quad|z| \rightarrow 1^{-}
$$

for any orthonormal basis $\left\{e_{n}\right\}$ of $\mathcal{D}_{\alpha}$.

Proof. Let $\left\{e_{n}\right\}$ be any orthonormal basis of $\mathcal{D}_{\alpha}$. From (2.2) and (2.5) we have

$$
\sum_{n} \overline{e_{n}^{\prime}(z)} e_{n}(z)=J_{z}^{\alpha}(z)=\int_{0}^{z} \frac{d \eta}{(1-\bar{z} \eta)^{2+\alpha}}=\frac{1-\left(1-|z|^{2}\right)^{1+\alpha}}{(1+\alpha) \bar{z}\left(1-|z|^{2}\right)^{1+\alpha}}
$$

and, since $\alpha \geq 0$, we obtain

$$
|z|\left(1-|z|^{2}\right)^{-1-\alpha} \leq(1+\alpha) \sum_{n}\left|e_{n}(z)\right|\left|e_{n}^{\prime}(z)\right|,
$$

which gives the result for $p=1$. If $1<p<2$, using Hölder's inequality

$$
\begin{aligned}
\frac{|z|}{1+\alpha}\left(1-|z|^{2}\right)^{-1-\alpha} & \leq \sum_{n}\left|e_{n}(z)\right|\left|e_{n}^{\prime}(z)\right| \\
& \leq\left(\sum_{n}\left|e_{n}(z)\right|^{p}\left|e_{n}^{\prime}(z)\right|^{2-p}\right)^{1 / p}\left(\sum_{n}\left|e_{n}^{\prime}(z)\right|^{2}\right)^{1 / p^{\prime}} \\
& \leq C\left(\sum_{n}\left|e_{n}(z)\right|^{p}\left|e_{n}^{\prime}(z)\right|^{2-p}\right)^{1 / p}\left(1-|z|^{2}\right)^{-(2+\alpha) / p^{\prime}}
\end{aligned}
$$

where the last inequality follows from (2.3) and (2.7). From here one obtains the corresponding inequality. The proof is complete.

We shall also use several times the following integral estimate (see [30]) that has become indispensable in this area of analysis.

Lemma B. Suppose $z \in \mathbb{D}, c \geq 0$ and $t>-1$. The integral

$$
I_{c, t}(z)=\int_{\mathbb{D}} \frac{\left(1-|w|^{2}\right)^{t}}{|1-\bar{w} z|^{2+t+c}} d A(w)
$$

is comparable to $\left(1-|z|^{2}\right)^{-c}$ if $c>0$, and to $\log \frac{1}{1-|z|^{2}}$ if $c=0$.

The useful inequality which appears below is from [13], and can be thought as a generalized version of the previous one. 
Lemma C. Let $s>-1, r, t>0$, and $r+t-s>2$. If $t<s+2<r$ then, for $a, z \in \mathbb{D}$, we have

$$
\int_{\mathbb{D}} \frac{\left(1-|w|^{2}\right)^{s}}{|1-\bar{w} z|^{r}|1-\bar{w} a|^{t}} d A(w) \leq C \frac{\left(1-|z|^{2}\right)^{2+s-r}}{|1-\bar{a} z|^{t}} .
$$

For $z \in \mathbb{D}$ and $r>0$, let

$$
D(z, r)=\{w \in \mathbb{D}: \beta(z, w)<r\}
$$

denote the hyperbolic disk with center $z$ and radius $r$. Here $\beta(z, w)$ is the Bergman or hyperbolic metric on $\mathbb{D}$.

We also need the concept of an $r$-lattice in the Bergman metric. Let $r>0$. A sequence $\left\{a_{k}\right\}$ of points in $\mathbb{D}$ is called an $r$-lattice, if the unit disk is covered by the Bergman metric disks $\left\{D_{k}:=D\left(a_{k}, r\right)\right\}$, and $\beta\left(a_{i}, a_{j}\right) \geq r / 2$ for all $i$ and $j$ with $i \neq j$. If $\left\{a_{k}\right\}$ is an $r$-lattice in $\mathbb{D}$, then it also has the following property: for any $R>0$ there exists a positive integer $N$ (depending on $r$ and $R$ ) such that every point in $\mathbb{D}$ belongs to at most $N$ sets in $\left\{D\left(a_{k}, R\right)\right\}$. There are elementary constructions of $r$-lattices in $\mathbb{D}$. See [30, Chapter 4$]$ for example.

\section{CASE $0<\alpha<1$.}

Before embarking on the proof of Theorem 1, some preliminary results of interest on their own must be proved.

3.1. A new class of spaces. In this subsection, we display several nesting properties of $X_{\alpha}^{p}$ and $B_{p}$ spaces. We offer a proof of (1.3), which gives under those restrictions an equivalent $B_{p}$-norm. It is worth noticing that equivalent and useful $B_{p}$-norms (see [1] and [7] for example) have been previously introduced for the study of operators on different spaces of analytic functions on $\mathbb{D}$. Also, our next result proves that $X_{\alpha}^{p} \subsetneq B_{p}$ if $0<\alpha<1$ and $p(1-\alpha) \geq 2$. In fact, $B_{p} \subset \mathcal{D}_{\alpha}$ if $p(1-\alpha)<2$, and this is no longer true when $p(1-\alpha) \geq 2$.

Proposition 3.1. Let $1<p<\infty$ and $\alpha \geq 0$. Then

(i) $X_{\alpha}^{p} \subset \mathcal{D}_{\alpha} \cap B_{p}$.

(ii) If $p<q$, then $X_{\alpha}^{p} \subset X_{\alpha}^{q}$.

(iii) If $0 \leq \alpha<\gamma$, then $X_{\alpha}^{p} \subset X_{\gamma}^{p}$.

(iv) Let $\alpha>0$. If $p(1-\alpha)<2$ then $X_{\alpha}^{p}=B_{p}$.

Proof. For $a \in \mathbb{D}$ fixed, let $D(a):=\left\{z:|z-a|<\frac{1-|a|}{2}\right\}$.

(i) If $g \in X_{\alpha}^{p}$, then the subharmonicity of $\left|g^{\prime}\right|^{2}$ together with the fact that $|1-\bar{w} z| \asymp\left(1-|w|^{2}\right)$ for $z \in D(w)$ implies that $g \in B_{p}$. Also, since 
$|1-\bar{w} z| \leq 2$

$$
\begin{aligned}
\|g\|_{X_{\alpha}^{p}}^{p} & \geq 2^{-(1+\alpha) p} \int_{\mathbb{D}}\left(\int_{\mathbb{D}}\left|g^{\prime}(z)\right|^{2} d A_{\alpha}(z)\right)^{p / 2}\left(1-|w|^{2}\right)^{p-2+\frac{\alpha p}{2}} d A(w) \\
& =C_{p, \alpha}\left\|g^{\prime}\right\|_{A_{\alpha}^{2}}^{p}
\end{aligned}
$$

This shows that $X_{\alpha}^{p} \subset \mathcal{D}_{\alpha}$ proving (i).

(ii) Assume that $g \in X_{\alpha}^{p}$. Fix $a \in \mathbb{D}$. If $w \in D(a)$, then $(1-|w|) \asymp(1-|a|)$ and $|1-\bar{w} z| \asymp|1-\bar{a} z|$ for $z \in \mathbb{D}$. So

$$
\begin{aligned}
\|g\|_{X_{\alpha}^{p}}^{p} & \geq \int_{D(a)}\left(\int_{\mathbb{D}} \frac{\left|g^{\prime}(z)\right|^{2} d A_{\alpha}(z)}{|1-\bar{w} z|^{2+2 \alpha}}\right)^{p / 2}\left(1-|w|^{2}\right)^{p-2+\alpha p / 2} d A(w) \\
& \geq C\left(1-|a|^{2}\right)^{p+\alpha p / 2}\left(\int_{\mathbb{D}} \frac{\left|g^{\prime}(z)\right|^{2} d A_{\alpha}(z)}{|1-\bar{a} z|^{2+2 \alpha}}\right)^{p / 2} .
\end{aligned}
$$

This gives

$$
\sup _{a \in \mathbb{D}}\left(1-|a|^{2}\right)^{2+\alpha} \int_{\mathbb{D}} \frac{\left|g^{\prime}(z)\right|^{2} d A_{\alpha}(z)}{|1-\bar{a} z|^{2+2 \alpha}}<\infty
$$

and it follows easily that $\|g\|_{X_{\alpha}^{q}}^{q} \leq C\|g\|_{X_{\alpha}^{p}}^{p}$ for $q>p$.

(iii) follows from the inequality $\sup _{z \in \mathbb{D}} \frac{\left(1-|z|^{2}\right)\left(1-|w|^{2}\right)}{|1-\bar{w} z|^{2}} \leq 1$.

(iv) The inclusion $X_{\alpha}^{p} \subset B_{p}$ follows from (i).

Conversely, suppose that $g \in B_{p}$. Assume first that $p>2$. Since $p \alpha>$ $p-2$, we can choose $\varepsilon>0$ with $p \alpha-(1+\varepsilon)(p-2)>0$. Then, using Hölder's inequality and Lemma B, we obtain

$$
\begin{aligned}
\left(\int_{\mathbb{D}} \frac{\left|g^{\prime}(z)\right|^{2} d A_{\alpha}(z)}{|1-\bar{w} z|^{2+2 \alpha}}\right)^{p / 2} & \leq\left(\int_{\mathbb{D}} \frac{\left|g^{\prime}(z)\right|^{p} d A_{t}(z)}{|1-\bar{w} z|^{2+p \alpha}}\right)\left(\int_{\mathbb{D}} \frac{\left(1-|z|^{2}\right)^{-1+\varepsilon} d A(z)}{|1-\bar{w} z|^{2}}\right)^{\frac{p-2}{2}} \\
& \leq C\left(\int_{\mathbb{D}} \frac{\left|g^{\prime}(z)\right|^{p} d A_{t}(z)}{|1-\bar{w} z|^{2+p \alpha}}\right)\left(1-|w|^{2}\right)^{(-1+\varepsilon) \frac{p-2}{2}}
\end{aligned}
$$

where $t=\frac{(1-\varepsilon)(p-2)+\alpha p}{2}$. This gives

$$
\| g-\left.g(0)\right|_{X_{\alpha}^{p}} ^{p} \leq C \int_{\mathbb{D}}\left|g^{\prime}(z)\right|^{p}\left(\int_{\mathbb{D}} \frac{\left(1-|w|^{2}\right)^{\beta}}{|1-\bar{w} z|^{2+p \alpha}} d A(w)\right) d A_{t}(z)
$$

with $\beta=\frac{(1+\varepsilon)(p-2)+\alpha p}{2}$. Note that the choice of $\varepsilon$ gives $p \alpha>\beta$, and therefore we can use Lemma B again in order to obtain

$$
\| g-g(0)||_{X_{\alpha}^{p}}^{p} \leq C \int_{\mathbb{D}}\left|g^{\prime}(z)\right|^{p}\left(1-|z|^{2}\right)^{t+\beta-p \alpha} d A(z)=C \int_{\mathbb{D}}\left|g^{\prime}(z)\right|^{p}\left(1-|z|^{2}\right)^{p-2} d A(z) .
$$


Now assume that $1<p \leq 2$. Fix an $r$-lattice $\left\{a_{n}\right\}$ with associated hyperbolic disks $\left\{D_{n}\right\}$. Then

$$
\begin{aligned}
\|g-g(0)\|_{X_{\alpha}^{p}}^{p} & \leq \int_{\mathbb{D}}\left(\sum_{n} \int_{D_{n}} \frac{\left|g^{\prime}(z)\right|^{2} d A_{\alpha}(z)}{|1-\bar{w} z|^{2+2 \alpha}}\right)^{p / 2}\left(1-|w|^{2}\right)^{p-2+\frac{\alpha p}{2}} d A(w) \\
& \asymp \int_{\mathbb{D}}\left(\sum_{n} \frac{\left(1-\left|a_{n}\right|^{2}\right)^{\alpha}}{\left|1-\bar{a}_{n} w\right|^{2+2 \alpha}} \int_{D_{n}}\left|g^{\prime}(z)\right|^{2} d A(z)\right)^{p / 2}\left(1-|w|^{2}\right)^{p-2+\frac{\alpha p}{2}} d A(w) \\
& \leq \int_{\mathbb{D}} \sum_{n} \frac{\left(1-\left|a_{n}\right|^{2}\right)^{p \alpha / 2}}{\left|1-\bar{a}_{n} w\right|^{p+p \alpha}}\left(\int_{D_{n}}\left|g^{\prime}(z)\right|^{2} d A(z)\right)^{p / 2}\left(1-|w|^{2}\right)^{p-2+\frac{\alpha p}{2}} d A(w) .
\end{aligned}
$$

Now, passing the sum outside the integral and using Lemma B we get

$$
\begin{aligned}
\|g-g(0)\|_{X_{\alpha}^{p}}^{p} & \leq \sum_{n}\left(1-\left|a_{n}\right|^{2}\right)^{p \alpha / 2}\left(\int_{D_{n}}\left|g^{\prime}(z)\right|^{2} d A(z)\right)^{p / 2} \int_{\mathbb{D}} \frac{\left(1-|w|^{2}\right)^{p-2+\frac{\alpha p}{2}} d A(w)}{\left|1-\bar{a}_{n} w\right|^{p+p \alpha}} \\
& \leq \sum_{n}\left(\int_{D_{n}}\left|g^{\prime}(z)\right|^{2} d A(z)\right)^{p / 2}<\infty,
\end{aligned}
$$

where the last step follows from Theorem 0 of [5] (see also [29]). This completes the proof.

3.2. Proof of Theorem 1. The sufficiency for the case $1<p \leq 2$, and the necessity for $2 \leq p<\infty$ is a byproduct of the following result, which also gives some information on the case $p(1-\alpha)>4$.

Proposition 3.2. Let $g \in H(\mathbb{D})$ and $\alpha \geq 0$.

(i) If $1<p \leq 2$ and $g \in X_{\alpha}^{p}$, then $T_{g} \in \mathcal{S}_{p}\left(\mathcal{D}_{\alpha}\right)$.

(ii) If $2 \leq p<\infty$ and $T_{g} \in S_{p}\left(\mathcal{D}_{\alpha}\right)$ then $g \in X_{\alpha}^{p}$.

Proof. Since

$$
\|g\|_{X_{\alpha}^{p}}^{p} \asymp \int_{\mathbb{D}}\left\|T_{g}\left(j_{z}^{\alpha}\right)\right\|_{\mathcal{D}_{\alpha}}^{p} d \lambda(z),
$$

the result follows directly from Proposition 2.2.

The necessity for $1<p<2$ follows from the next Proposition and part (iv) of Proposition 3.1.

Proposition 3.3. Let $0 \leq \alpha<1$ and $g \in H(\mathbb{D})$. Then

(i) If $1<p<2$ and $T_{g} \in \mathcal{S}_{p}\left(\mathcal{D}_{\alpha}\right)$, then $g \in B_{p}$.

(ii) If $T_{g} \in \mathcal{S}_{1}\left(\mathcal{D}_{\alpha}\right)$, then $g$ is constant.

Proof. Let $1 \leq p<2$, and assume that $T_{g} \in \mathcal{S}_{p}\left(\mathcal{D}_{\alpha}\right)$. Then the positive operator $T_{g}^{*} T_{g}$ belongs to $\mathcal{S}_{p / 2}\left(\mathcal{D}_{\alpha}\right)$. Without loss of generality we may assume that $g^{\prime} \neq 0$. Suppose

$$
T_{g}^{*} T_{g} f=\sum_{n} \lambda_{n}\left\langle f, e_{n}\right\rangle e_{n}
$$


is the canonical decomposition of $T_{g}^{*} T_{g}$. Then not only is $\left\{e_{n}\right\}$ an orthonormal set, it is also an orthonormal basis. Indeed, if there is an unit vector $e \in \mathcal{D}_{\alpha}$ such that $e \perp e_{n}$ for all $n \geq 1$, then

$$
\int_{\mathbb{D}}\left|g^{\prime}(z)\right|^{2}|e(z)|^{2} d A_{\alpha}(z)=\left\|T_{g} e\right\|_{\mathcal{D}_{\alpha}}^{2}=\left\langle T_{g}^{*} T_{g} e, e\right\rangle_{\mathcal{D}_{\alpha}}=0
$$

because $T_{g}^{*} T_{g}$ is a linear combination of the vectors $e_{n}$. This would give $g^{\prime} \equiv 0$.

Since $\left\{e_{n}\right\}$ is an orthonormal basis of $\mathcal{D}_{\alpha}$, then by Lemma 2.3

$$
\begin{aligned}
\int_{\mathbb{D}}\left|g^{\prime}(z)\right|^{p} & \left(1-|z|^{2}\right)^{p-2} d A(z) \\
& \leq C \int_{\mathbb{D}}\left|g^{\prime}(z)\right|^{p}\left(\sum_{n}\left|e_{n}(z)\right|^{p}\left|e_{n}^{\prime}(z)\right|^{2-p}\right) d A_{\alpha}(z) \\
& \leq C \sum_{n}\left(\int_{\mathbb{D}}\left|g^{\prime}(z)\right|^{2}\left|e_{n}(z)\right|^{2} d A_{\alpha}(z)\right)^{p / 2} \\
& =C \sum_{n}\left\langle T_{g}^{*} T_{g} e_{n}, e_{n}\right\rangle_{\mathcal{D}_{\alpha}}^{p / 2}=C \sum_{n} \lambda_{n}^{p / 2}=C\left\|T_{g}^{*} T_{g}\right\|_{\mathcal{S}_{p / 2}^{p / 2}}^{p},
\end{aligned}
$$

which finishes the proof of (i). Furthermore, if $T_{g} \in \mathcal{S}_{1}\left(\mathcal{D}_{\alpha}\right)$, then (3.2) says that

$$
\int_{\mathbb{D}}\left|g^{\prime}(z)\right|\left(1-|z|^{2}\right)^{-1} d A(z)<\infty
$$

which implies that $g$ is constant. This completes the proof.

The remaining part of the proof is more involved. It will be splitted in two cases.

Sufficiency. Case $\mathbf{2}<\mathbf{p} \leq \mathbf{4}$. Let $\left\{e_{n}\right\}$ be any orthonormal set in $\mathcal{D}_{\alpha}$. Then

$$
\sum_{n}\left\|T_{g} e_{n}\right\|_{\mathcal{D}_{\alpha}}^{p}=\sum_{n}\left(\int_{\mathbb{D}}\left|g^{\prime}(z)\right|^{2}\left|e_{n}(z)\right|^{2} d A_{\alpha}(z)\right)^{p / 2} \leq C\left(I_{1}+I_{2}\right),
$$

with

$$
I_{1}=\sum_{n}\left|e_{n}(0)\right|^{p}\left(\int_{\mathbb{D}}\left|g^{\prime}(z)\right|^{2} d A_{\alpha}(z)\right)^{p / 2}
$$

and

$$
I_{2}=\sum_{n}\left(\int_{\mathbb{D}}\left|g^{\prime}(z)\right|^{2}\left|e_{n}^{2}(z)-e_{n}^{2}(0)\right| d A_{\alpha}(z)\right)^{p / 2} .
$$

Since $g \in X_{\alpha}^{p} \subset \mathcal{D}_{\alpha}$ by Lemma 3.1 and $\left|e_{n}(0)\right| \leq 1$, we clearly have

$$
I_{1} \leq\|g\|_{\mathcal{D}_{\alpha}}^{p} \sum_{n}\left|e_{n}(0)\right|^{2} \leq\|g\|_{\mathcal{D}_{\alpha}}^{p}\left\|K_{0}^{\alpha}\right\|_{\mathcal{D}_{\alpha}}^{2} \leq C\|g\|_{X_{\alpha}^{p}}^{p} .
$$


In order to deal with the term $I_{2}$, note first that $e_{n}^{2} \in \mathcal{D}_{1+2 \alpha}$ because for any $f \in \mathcal{D}_{\alpha}$,

$$
|f(z)|^{2} \leq C \frac{\|f\|_{\mathcal{D}_{\alpha}}^{2}}{(1-|z|)^{\alpha}}, \quad z \in \mathbb{D}
$$

So from the reproducing formula for $\mathcal{D}_{1+2 \alpha}$ we deduce

$$
\left|e_{n}^{2}(z)-e_{n}^{2}(0)\right| \leq C \int_{\mathbb{D}} \frac{\left|e_{n}(w)\right|\left|e_{n}^{\prime}(w)\right|}{|1-\bar{w} z|^{2+2 \alpha}} d A_{1+2 \alpha}(w) .
$$

Therefore, if we use the notation

$$
J_{n}(g):=\int_{\mathbb{D}}\left|g^{\prime}(z)\right|^{2}\left|e_{n}^{2}(z)-e_{n}^{2}(0)\right| d A_{\alpha}(z),
$$

Fubini's theorem and Hölder's inequality yields

$$
\begin{aligned}
J_{n}(g)^{p / 2} & \leq C\left(\int_{\mathbb{D}}\left|e_{n}(w)\right|\left|e_{n}^{\prime}(w)\right|\left[\int_{\mathbb{D}} \frac{\left|g^{\prime}(z)\right|^{2} d A_{\alpha}(z)}{|1-\bar{w} z|^{2+2 \alpha}}\right] d A_{1+2 \alpha}(w)\right)^{p / 2} \\
& \leq C \int_{\mathbb{D}}\left|e_{n}(w)\right|^{\frac{p}{2}}\left|e_{n}^{\prime}(w)\right|^{2-\frac{p}{2}}\left(\int_{\mathbb{D}} \frac{\left|g^{\prime}(z)\right|^{2} d A_{\alpha}(z)}{|1-\bar{w} z|^{2+2 \alpha}}\right)^{p / 2} d A_{(1+\alpha) \frac{p}{2}+\alpha}(w) .
\end{aligned}
$$

Then, if $p=4$, it follows from $(2.3)$ and the fact that $\left\|K_{w}^{\alpha}\right\|_{\mathcal{D}_{\alpha}}^{2} \asymp\left(1-|w|^{2}\right)^{-\alpha}$ that

$$
\begin{aligned}
I_{2}=\sum_{n} J_{n}(g)^{2} \leq & C \int_{\mathbb{D}}\left\|K_{w}^{\alpha}\right\|_{\mathcal{D}_{\alpha}}^{2}\left(\int_{\mathbb{D}} \frac{\left|g^{\prime}(z)\right|^{2} d A_{\alpha}(z)}{|1-\bar{w} z|^{2+2 \alpha}}\right)^{2} d A_{(1+\alpha) 2+\alpha}(w) \\
& \leq C \int_{\mathbb{D}}\left(\int_{\mathbb{D}} \frac{\left|g^{\prime}(z)\right|^{2} d A_{\alpha}(z)}{|1-\bar{w} z|^{2+2 \alpha}}\right)^{2} d A_{(1+\alpha) 2}(w)=\|g\|_{X_{\alpha}^{4}}^{4} .
\end{aligned}
$$

Now, if $2<p<4$, notice that Hölder's inequality with exponent $4 / p>1$ and (2.3) yield

$$
\begin{aligned}
\sum_{n}\left|e_{n}(w)\right|^{\frac{p}{2}}\left|e_{n}^{\prime}(w)\right|^{2-\frac{p}{2}} & \leq\left(\sum_{n}\left|e_{n}(w)\right|^{2}\right)^{p / 4}\left(\sum_{n}\left|e_{n}^{\prime}(w)\right|^{2}\right)^{1-\frac{p}{4}} \\
& \leq\left\|K_{w}^{\alpha}\right\|_{\mathcal{D}_{\alpha}}^{p / 2}\left\|J_{w}^{\alpha}\right\|_{\mathcal{D}_{\alpha}}^{\frac{(4-p)}{2}} .
\end{aligned}
$$

This together with the fact that for $\alpha>0$ we have $\left\|K_{w}^{\alpha}\right\|_{\mathcal{D}_{\alpha}}^{2} \asymp\left(1-|w|^{2}\right)^{-\alpha}$ and $\left\|J_{w}^{\alpha}\right\|_{\mathcal{D}_{\alpha}}^{2}=\left(1-|w|^{2}\right)^{-(2+\alpha)}$, gives

$$
\begin{aligned}
I_{2} & =\sum_{n} J_{n}(g)^{p / 2} \\
& \leq C \int_{\mathbb{D}} \sum_{n}\left|e_{n}(w)\right|^{\frac{p}{2}}\left|e_{n}^{\prime}(w)\right|^{2-\frac{p}{2}}\left(\int_{\mathbb{D}} \frac{\left|g^{\prime}(z)\right|^{2} d A_{\alpha}(z)}{|1-\bar{w} z|^{2+2 \alpha}}\right)^{p / 2} d A_{(1+\alpha) \frac{p}{2}+\alpha}(w) \\
& \leq C \int_{\mathbb{D}}\left\|K_{w}^{\alpha}\right\|_{\mathcal{D}_{\alpha}}^{p / 2}\left\|J_{w}^{\alpha}\right\|_{\mathcal{D}_{\alpha}^{2}}^{\frac{4-p}{2}}\left(\int_{\mathbb{D}} \frac{\left|g^{\prime}(z)\right|^{2} d A_{\alpha}(z)}{|1-\bar{w} z|^{2+2 \alpha}}\right)^{p / 2} d A_{(1+\alpha) \frac{p}{2}+\alpha}(w) \\
& \leq C \int_{\mathbb{D}}\left(\int_{\mathbb{D}} \frac{\left|g^{\prime}(z)\right|^{2} d A_{\alpha}(z)}{|1-\bar{w} z|^{2+2 \alpha}}\right)^{p / 2} d A_{p-2+\alpha \frac{p}{2}}(w)=C\|g\|_{X_{\alpha}^{p}}^{p} .
\end{aligned}
$$


Since $g \in X_{\alpha}^{p}$ combining the estimates for $I_{2}$ and $I_{1}$ we obtain that

$$
\sum_{n}\left\|T_{g} e_{n}\right\|_{\mathcal{D}_{\alpha}}^{p} \leq C<\infty
$$

Thus, by [30, Theorem 1.33], the operator $T_{g}$ belongs to $\mathcal{S}_{p}\left(\mathcal{D}_{\alpha}\right)$.

Sufficiency. Case $4<\mathrm{p}<\infty$ and $\mathrm{p}(1-\alpha)<4$. Proceeding as before we get

$$
\begin{aligned}
J_{n}(g)^{p / 2} & \leq C\left(\int_{\mathbb{D}}\left|e_{n}(w)\right|\left|e_{n}^{\prime}(w)\right|\left[\int_{\mathbb{D}} \frac{\left|g^{\prime}(z)\right|^{2} d A_{\alpha}(z)}{|1-\bar{w} z|^{2+2 \alpha}}\right] d A_{1+2 \alpha}(w)\right)^{p / 2} \\
& \leq C\left(\int_{\mathbb{D}}\left|e_{n}(w)\right|^{2} S_{\alpha} g(w)^{2} d A_{2+\alpha}(w)\right)^{p / 4}
\end{aligned}
$$

where

$$
S_{\alpha} g(w)=\left(1-|w|^{2}\right)^{\alpha} \int_{\mathbb{D}} \frac{\left|g^{\prime}(z)\right|^{2} d A_{\alpha}(z)}{|1-\bar{w} z|^{2+2 \alpha}} .
$$

Since $\alpha>0, p>4$ and $p(1-\alpha)<4$, Hölder's inequality implies that $\left\|S_{\alpha} g\right\|_{L^{2}\left(\mathbb{D}, d A_{2+\alpha}\right)}^{2} \leq C\|g\|_{X_{\alpha}^{p}}^{4}$, and therefore we can assume that $e_{n}(0)=0$. Note that for $\beta \geq \alpha$ we have

$$
\left|e_{n}(w)\right|=\left|e_{n}(w)-e_{n}(0)\right| \leq C \int_{\mathbb{D}} \frac{\left|e_{n}^{\prime}(\zeta)\right|}{|1-\bar{\zeta} w|^{1+\beta}} d A_{\beta}(\zeta) .
$$

This follows from the reproducing formula for $\mathcal{D}_{\beta}$ and the fact that $\mathcal{D}_{\alpha} \subset \mathcal{D}_{\beta}$ if $\alpha \leq \beta$. Since $p \alpha>p-4$, we can take $\varepsilon>0$ so that

$$
\alpha p-3 \varepsilon p>p-4 \text {. }
$$

Now, choose

$$
\beta>\max \left\{1+\frac{p \varepsilon}{2}, \frac{\alpha p-3 \varepsilon p}{p-4}, \alpha-\varepsilon+\frac{4}{p}\right\} .
$$

An application of Cauchy-Schwarz inequality and Lemma B, together with (3.5) gives

$$
\left|e_{n}(w)\right|^{2} \leq C\left(\int_{\mathbb{D}} \frac{\left|e_{n}^{\prime}(\zeta)\right|^{2}}{|1-\bar{\zeta} w|^{\beta}} d A_{\beta+\varepsilon}(\zeta)\right)\left(1-|w|^{2}\right)^{-\varepsilon}
$$

The use of (3.6), Fubini's theorem and Hölder's inequality give

$$
\begin{aligned}
J_{n}(g)^{p / 2} & \leq C\left(\int_{\mathbb{D}}\left|e_{n}^{\prime}(\zeta)\right|^{2}\left(\int_{\mathbb{D}} \frac{S_{\alpha} g(w)^{2}}{|1-\bar{\zeta} w|^{\beta}} d A_{2+\alpha-\varepsilon}(w)\right) d A_{\beta+\varepsilon}(\zeta)\right)^{p / 4} \\
& \leq C \int_{\mathbb{D}}\left|e_{n}^{\prime}(\zeta)\right|^{2}\left(\int_{\mathbb{D}} \frac{S_{\alpha} g(w)^{2}}{|1-\bar{\zeta} w|^{\beta}} d A_{2+\alpha-\varepsilon}(w)\right)^{p / 4} d A_{(\beta+\varepsilon) p / 4+\alpha\left(1-\frac{p}{4}\right)}(\zeta) .
\end{aligned}
$$

Thus, by (2.3)

$$
\sum_{n} J_{n}(g)^{p / 2} \leq C \int_{\mathbb{D}}\left(\int_{\mathbb{D}} \frac{S_{\alpha} g(w)^{2}}{|1-\bar{\zeta} w|^{\beta}} d A_{2+\alpha-\varepsilon}(w)\right)^{p / 4} d A_{-2+(\beta+\varepsilon-\alpha) \frac{p}{4}}(\zeta) .
$$


Let

$$
\gamma=\frac{-2 p+8+\alpha p-3 \varepsilon p}{p-4}=-2+\frac{\alpha p-3 \varepsilon p}{p-4} .
$$

By (3.4), we have $\gamma>-1$. Now, using Hölder's inequality, (3.5) and Lemma B we obtain

$$
\begin{aligned}
\int_{\mathbb{D}} & \frac{S_{\alpha} g(w)^{2}}{|1-\bar{\zeta} w|^{\beta}} d A_{2+\alpha-\varepsilon}(w) \\
& \leq\left(\int_{\mathbb{D}} \frac{S_{\alpha} g(w)^{p / 2}}{|1-\bar{\zeta} w|^{\beta}} d A_{p-2+\varepsilon \frac{p}{2}}(w)\right)^{\frac{4}{p}}\left(\int_{\mathbb{D}} \frac{d A_{\gamma}(w)}{|1-\bar{\zeta} w|^{\beta}}\right)^{\frac{p-4}{p}} \\
& \leq\left(\int_{\mathbb{D}} \frac{S_{\alpha} g(w)^{p / 2}}{|1-\bar{\zeta} w|^{\beta}} d A_{p-2+\varepsilon \frac{p}{2}}(w)\right)^{\frac{4}{p}}\left(\left(1-|\zeta|^{2}\right)^{\gamma+2-\beta}\right)^{\frac{p-4}{p}} .
\end{aligned}
$$

Putting this into (3.7), Fubini's theorem an Lemma B yields

$$
\begin{aligned}
\sum_{n} J_{n}(g)^{p / 2} & \leq C \int_{\mathbb{D}}\left(\int_{\mathbb{D}} \frac{S_{\alpha} g(w)^{p / 2}}{|1-\bar{\zeta} w|^{\beta}} d A_{p-2+\varepsilon \frac{p}{2}}(w)\right)\left(1-|\zeta|^{2}\right)^{\beta-2-\varepsilon \frac{p}{2}} d A(\zeta) \\
& =C \int_{\mathbb{D}} S_{\alpha} g(w)^{p / 2}\left(\int_{\mathbb{D}} \frac{\left(1-|\zeta|^{2}\right)^{\beta-2-\varepsilon \frac{p}{2}}}{|1-\bar{\zeta} w|^{\beta}} d A(\zeta)\right) d A_{p-2+\varepsilon \frac{p}{2}(w)} \\
& \leq C \int_{\mathbb{D}} S_{\alpha} g(w)^{p / 2} d A_{p-2}(w)=C|| g \|_{X_{\alpha}^{p}}^{p}
\end{aligned}
$$

3.3. The open case. In relation with the open case $p(1-\alpha) \geq 4$, we provide a result which can be proved following the lines of the proof of Theorem 1 (case $p>4$ ), and therefore the proof will be omitted.

Proposition 3.4. Let $0<\alpha, p \geq 2$ and $g \in H(\mathbb{D})$. If $g \in X_{\alpha-\varepsilon}^{p}$ for some $\varepsilon \in(0, \alpha)$, then $T_{g} \in \mathcal{S}_{p}\left(\mathcal{D}_{\alpha}\right)$.

Obviously, $X_{\alpha-\varepsilon}^{p} \subsetneq X_{\alpha}^{p}$ if $(1-\alpha) p \geq 2$ (see Lemma 4.1 below), so Proposition 3.4 gives a sufficient but not necessary condition for $T_{g} \in \mathcal{S}_{p}\left(\mathcal{D}_{\alpha}\right)$, $(1-\alpha) p \geq 2$. However, if $\alpha>0$ and $1<p<\infty$, those techniques which will be developed in the proof of Lemma 4.2, together with Lemma C, imply that for any $\beta>0$,

$$
\left\|T_{g_{a}}\right\|_{\mathcal{S}_{p}\left(\mathcal{D}_{\alpha}\right)} \asymp\left\|g_{a}\right\|_{B_{p}} \asymp\left\|g_{a}\right\|_{X_{\beta}^{p}},
$$

where $g_{a}(z)=(1-\bar{a} z)^{-\gamma}, \gamma>0$. In particular, the previous result gives the right growth for this family of functions.

\section{Schatten Classes of $T_{g}$ On the Classical Dirichlet space}

\subsection{Case $\mathrm{p} \leq \mathbf{2}$.}

Proof of Theorem 2. Since $\mathcal{S}_{p}(\mathcal{D}) \subset \mathcal{S}_{1}(\mathcal{D})$ for $0<p \leq 1$, the result follows from part (ii) of Proposition 3.3. 
Proof of Theorem 3. Part (a) follows from part (i) of Proposition 3.3, and part (c) is deduced in Proposition 3.2. In order to prove part (b), assume that $1<p<2$. Then, for all orthonormal sets $\left\{e_{n}\right\}$ of $\mathcal{D}$, we have

$$
\begin{aligned}
\sum_{n}\left|\left\langle T_{g} e_{n}, e_{n}\right\rangle_{\mathcal{D}}\right|^{p} & \leq \sum_{n}\left(\int_{\mathbb{D}}\left|g^{\prime}(z)\right|\left|e_{n}(z)\right|\left|e_{n}^{\prime}(z)\right| d A(z)\right)^{p} \\
& \leq \sum_{n}\left(\int_{\mathbb{D}}\left|g^{\prime}(z)\right|^{p}\left|e_{n}(z)\right|^{p}\left|e_{n}^{\prime}(z)\right|^{2-p} d A(z)\right)\left(\int_{\mathbb{D}}\left|e_{n}^{\prime}(z)\right|^{2} d A(z)\right)^{p / p^{\prime}} \\
& \leq \int_{\mathbb{D}}\left|g^{\prime}(z)\right|^{p}\left(\sum_{n}\left|e_{n}(z)\right|^{p}\left|e_{n}^{\prime}(z)\right|^{2-p}\right) d A(z) \\
& \leq \int_{\mathbb{D}}\left|g^{\prime}(z)\right|^{p}\left(\sum_{n}\left|e_{n}(z)\right|^{2}\right)^{p / 2}\left(\sum_{n}\left|e_{n}^{\prime}(z)\right|^{2}\right)^{1-p / 2} d A(z) \\
& \leq C \int_{\mathbb{D}}\left|g^{\prime}(z)\right|^{p}\left(\log \frac{e}{1-|z|}\right)^{p / 2}\left(1-|z|^{2}\right)^{p-2} d A(z) .
\end{aligned}
$$

Thus, by [30, Theorem 1.27], we deduce that $T_{g} \in \mathcal{S}_{p}(\mathcal{D})$ with $\left\|T_{g}\right\|_{\mathcal{S}_{p}} \leq$ $C\|g\|_{B_{p} \log ^{p / 2}}$.

4.2. Testing functions for Schatten classes. Our next goal consists of proving that Theorem 3 gives the correct behavior of $\left\|T_{g}\right\|_{\mathcal{S}_{p}}, 1<p<2$, at least for some families of functions. For the beginning, we deal with monomials.

Lemma 4.1. Asumme that $0 \leq \alpha<1$ and $1<p<\infty$. Let $g_{j}(z)=z^{j}$, $j=1,2,3 \ldots$ Then

$$
\begin{aligned}
\left\|T_{g_{j}}\right\|_{\mathcal{S}_{p}\left(\mathcal{D}_{\alpha}\right)} \asymp\left\|g_{j}\right\|_{X_{\alpha}^{p}} \asymp\left\{\begin{array}{lll}
j^{\frac{1}{p}} & \text { if } & (1-\alpha) p<2, \\
(j \log (j+1))^{\frac{1}{p}} & \text { if } & (1-\alpha) p=2, \\
j \frac{1-\alpha}{2} & \text { if } & (1-\alpha) p \geq 2,
\end{array}\right. \\
\left\|g_{j}\right\|_{B_{p, \log ^{p / 2}}} \asymp j^{1 / p}(\log (j+1))^{1 / 2},
\end{aligned}
$$

and

$$
\left\|g_{j}\right\|_{B_{p}} \asymp j^{1 / p} \text {. }
$$

Proof. We shall use the inner product in $\mathcal{D}_{\alpha}$ given by

$$
\langle f, g\rangle=\sum_{k=0}^{\infty}(k+1)^{1-\alpha} a_{k} \overline{b_{k}},
$$

for $f(z)=\sum_{n=0}^{\infty} a_{n} z^{n}$, and $g(z)=\sum_{n=0}^{\infty} b_{n} z^{n}$. We note that

$$
T_{g_{j}}(f)(z)=j \sum_{k=0}^{\infty} \frac{a_{k}}{k+j} z^{k+j}=j \sum_{n=j}^{\infty} \frac{a_{n-j}}{n} z^{n} .
$$


Now, if $\sigma_{n}(z)=\frac{z^{n}}{(n+1)^{\frac{1-\alpha}{2}}}, n \in \mathbb{N}$, we have that $\left\{\sigma_{n}\right\}_{n=0}^{\infty}$ is an orthonomal basis of $\mathcal{D}_{\alpha}$, and furthermore

$$
\sum_{n=j}^{\infty} \frac{a_{n-j}}{n} z^{n}=\sum_{n=j}^{\infty} \frac{a_{n-j}(n+1)^{\frac{1-\alpha}{2}}}{n} \sigma_{n}=\sum_{n=j}^{\infty} \frac{(n+1)^{\frac{1-\alpha}{2}}}{n(n-j+1)^{\frac{1-\alpha}{2}}}\left\langle f, \sigma_{n-j}\right\rangle \sigma_{n} .
$$

That is, the singular values of the integration operator $T_{g_{j}}$ are $\left\{\frac{j(n+1)^{\frac{1-\alpha}{2}}}{n(n-j+1)^{\frac{1-\alpha}{2}}}\right\}_{n=j}^{\infty}$. Consequently,

$$
\left\|T_{g_{j}}\right\|_{\mathcal{S}_{p}}^{p} \asymp j^{p} \sum_{n=j}^{\infty} \frac{1}{\left(n^{1+\alpha}(n-j+1)^{1-\alpha}\right)^{p / 2}} .
$$

On the other hand,

$$
\begin{aligned}
\left\|g_{j}\right\|_{X_{\alpha}^{p}}^{p} & =j^{p} \int_{\mathbb{D}}\left(\int_{\mathbb{D}} \frac{|\zeta|^{2(j-1)}}{|1-\bar{z} \zeta|^{2+2 \alpha}} d A_{\alpha}(\zeta)\right)^{\frac{p}{2}}\left(1-|z|^{2}\right)^{p-2+\frac{p \alpha}{2}} d A(z) . \\
& \asymp j^{p} \int_{\mathbb{D}}\left(\int_{0}^{1} \frac{r^{2 j-1}(1-r)^{\alpha}}{(1-r|z|)^{1+2 \alpha}} d r\right)^{\frac{p}{2}}\left(1-|z|^{2}\right)^{p-2+\frac{p \alpha}{2}} d A(z) \\
& \asymp j^{p} \int_{\mathbb{D}}\left(\sum_{m=0}^{\infty} \frac{(m+1)^{2 \alpha}|z|^{m}}{(2 j+m)^{1+\alpha}}\right)^{\frac{p}{2}}\left(1-|z|^{2}\right)^{p-2+\frac{p \alpha}{2}} d A(z) \\
& \asymp j^{p} \int_{0}^{1}\left(\sum_{m=0}^{\infty} \frac{(m+1)^{2 \alpha} s^{m}}{(2 j+m)^{1+\alpha}}\right)^{\frac{p}{2}}\left(1-s^{2}\right)^{p-2+\frac{p \alpha}{2}} d s .
\end{aligned}
$$

At this point, we use [12, Theorem 1] to obtain

$$
\begin{aligned}
\left\|g_{j}\right\|_{X_{\alpha}^{p}}^{p} & \asymp j^{p} \sum_{n=0}^{\infty} \frac{1}{2^{n\left(p-1+\frac{p \alpha}{2}\right)}}\left(\sum_{m \in I(n)} \frac{(m+1)^{2 \alpha}}{(2 j+m)^{1+\alpha}}\right)^{p / 2} \\
& \asymp j^{p} \sum_{n=0}^{\infty} \frac{2^{n}}{\left[2^{n(1-\alpha)}\left(2^{n}+2 j\right)^{1+\alpha}\right]^{p / 2}} \\
& \asymp j^{p} \sum_{n=0}^{\infty}\left(\sum_{m \in I(n)} \frac{1}{\left[(m+1)^{(1-\alpha)}(m+j)^{(1+\alpha)}\right]^{p / 2}}\right) \\
& \asymp j^{p} \sum_{n=0}^{\infty} \frac{1}{\left[(n+1)^{(1-\alpha)}(n+j)^{(1+\alpha)}\right]^{p / 2}} \\
& \asymp j^{p} \sum_{n=j}^{\infty} \frac{1}{\left[n^{(1+\alpha)}(n-j+1)^{(1-\alpha)}\right]^{p / 2}},
\end{aligned}
$$

which together with (4.4) gives the first equivalence in (4.1). The second equivalence in (4.1) follows from an straightforward calculation according to those values of $p$ and $\alpha$. 
Now we prove (4.2),

$$
\begin{aligned}
\left\|g_{j}\right\|_{B_{p, \log p / 2}}^{p} & =j^{p} \int_{0}^{1} r^{(j-1) p+1}(1-r)^{p-2}\left(\log \frac{e}{1-r}\right)^{p / 2} d r \\
& \geq j^{p} \int_{1-\frac{1}{j+1}}^{1} r^{(j-1) p+1}(1-r)^{p-2}\left(\log \frac{e}{1-r}\right)^{p / 2} d r \\
& \asymp j^{p} \int_{1-\frac{1}{j+1}}^{1}(1-r)^{p-2}\left(\log \frac{e}{1-r}\right)^{p / 2} d r \\
& \asymp j(\log (j+1))^{p / 2},
\end{aligned}
$$

where in the last step we have used that $\omega(r)=(1-r)^{p-2}\left(\log \frac{e}{1-r}\right)^{p / 2}$ is an admissible weight with distortion function equivalent to $(1-r)$ (see $[15, \mathrm{p}$. 11]). Now, bearing in mind the properties of the Beta function,

$$
\begin{aligned}
& j^{p} \int_{0}^{1-\frac{1}{j+1}} r^{(j-1) p+1}(1-r)^{p-2}\left(\log \frac{e}{1-r}\right)^{p / 2} d r \\
& \leq C j^{p}(\log (j+1))^{p / 2} \int_{0}^{1-\frac{1}{j+1}} r^{(j-1) p+1}(1-r)^{p-2} d r \\
& \leq C j^{p}(\log (j+1))^{p / 2} \int_{0}^{1} r^{(j-1) p+1}(1-r)^{p-2} d r \\
& \asymp j(\log (j+1))^{p / 2},
\end{aligned}
$$

so we get (4.2). The equivalence (4.3) can be proved analogously. This finishes the proof.

Next, for each $a \in \mathbb{D}$ and $\gamma>0$, consider the functions $g_{a}(z)=(1-\bar{a} z)^{-\gamma}$.

Lemma 4.2. Assume that $p>1$ and $\gamma>0$. Then

$$
\begin{gathered}
\left\|g_{a}\right\|_{B_{p}} \asymp\left(1-|a|^{2}\right)^{-\gamma}, \\
\left\|g_{a}\right\|_{X_{0}^{p}} \asymp(1-|a|)^{-\gamma}\left(\log \frac{e}{1-|a|}\right)^{1 / p}, \quad|a| \rightarrow 1^{-},
\end{gathered}
$$

and

$$
\left\|T_{g_{a}}\right\|_{\mathcal{S}_{p}(\mathcal{D})} \asymp\left\|g_{a}\right\|_{B_{p, \log p / 2}} \asymp\left(1-|a|^{2}\right)^{-\gamma}\left(\log \frac{e}{1-|a|^{2}}\right)^{1 / 2} .
$$


Proof. A simple use of Lemma B implies (4.5). Since $|1-\bar{w} a| \asymp|1-\bar{w} z|$ for any $z \in D(a)=\left\{z:|z-a|<\frac{1-|a|}{2}\right\}$, it follows that

$$
\begin{aligned}
\int_{\mathbb{D}} \frac{d A(z)}{|1-\bar{w} z|^{2}|1-\bar{a} z|^{2 \gamma+2}} & \geq \int_{D(a)} \frac{d A(z)}{|1-\bar{w} z|^{2}|1-\bar{a} z|^{2 \gamma+2}} \\
& \geq \frac{C}{(1-|a|)^{2 \gamma+2}} \int_{D_{a}} \frac{d A(z)}{|1-\bar{w} z|^{2}} \\
& \geq \frac{C}{(1-|a|)^{2 \gamma}|1-\bar{w} a|^{2}} .
\end{aligned}
$$

Therefore, joining this and Lemma B,

$$
\begin{aligned}
\left\|g_{a}\right\|_{X_{0}^{p}}^{p}= & |a \gamma|^{p} \int_{\mathbb{D}}\left(\int_{\mathbb{D}} \frac{d A(z)}{|1-\bar{w} z|^{2}|1-\bar{a} z|^{2 \gamma+2}}\right)^{p / 2}\left(1-|w|^{2}\right)^{p-2} d A(w) \\
& \geq C \frac{C}{(1-|a|)^{p \gamma}} \int_{\mathbb{D}} \frac{\left(1-|w|^{2}\right)^{p-2} d A(w)}{|1-\bar{w} a|^{p}} \\
& \asymp(1-|a|)^{-p \gamma}\left(\log \frac{e}{1-|a|}\right) .
\end{aligned}
$$

On the other hand, taking $0<\varepsilon<\min (1,2(p-1) / p)$, and bearing in mind Lemma C,

$$
\begin{aligned}
\int_{\mathbb{D}} \frac{d A(z)}{|1-\bar{w} z|^{2}|1-\bar{a} z|^{2 \gamma+2}} & \leq(1-|w|)^{-\varepsilon} \int_{\mathbb{D}} \frac{d A(z)}{|1-\bar{w} z|^{2-\varepsilon}|1-\bar{a} z|^{2 \gamma+2}} \\
& \leq C \frac{\left(1-|w|^{2}\right)^{-\varepsilon}}{(1-|a|)^{2 \gamma}|1-\bar{w} a|^{2-\varepsilon}}
\end{aligned}
$$

So, an application of Lemma B gives

$$
\begin{aligned}
\left\|g_{a}\right\|_{X_{0}^{p}}^{p} & \leq \frac{C}{(1-|a|)^{p \gamma}} \int_{\mathbb{D}} \frac{\left(1-|w|^{2}\right)^{p-2-\varepsilon p / 2} d A(w)}{|1-\bar{w} a|^{p-\varepsilon \frac{p}{2}}} \\
& \asymp(1-|a|)^{-p \gamma}\left(\log \frac{e}{1-|a|}\right), \quad|a| \rightarrow 1^{-} .
\end{aligned}
$$

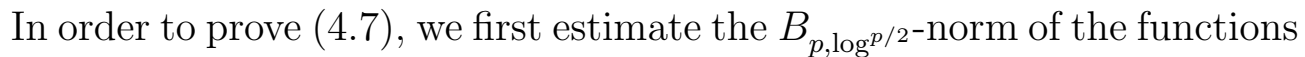
$g_{a}(z)=(1-\bar{a} z)^{-\gamma}$. Take $a \in \mathbb{D}$ with $|a| \geq 1 / 2$.

$$
\begin{aligned}
\left\|g_{a}\right\|_{B_{p, \log }^{p / 2}}^{p} & \asymp \int_{0}^{1} \frac{(1-s)^{p-2}\left(\log \frac{e}{1-s}\right)^{p / 2} d s}{(1-|a| s)^{p \gamma+p-1}} \\
& =\int_{0}^{|a|} \frac{(1-s)^{p-2}\left(\log \frac{e}{1-s}\right)^{p / 2} d s}{(1-|a| s)^{p \gamma+p-1}}+\int_{|a|}^{1} \frac{(1-s)^{p-2}\left(\log \frac{e}{1-s}\right)^{p / 2} d s}{(1-|a| s)^{p \gamma+p-1}} \\
& =I_{1}(|a|)+I_{2}(|a|) .
\end{aligned}
$$


Since $(1-s)^{p-2}\left(\log \frac{e}{1-s}\right)^{p / 2}$ is an admissible weight

$$
\begin{aligned}
I_{2}(|a|) & \asymp \frac{1}{(1-|a|)^{p \gamma+p-1}} \int_{|a|}^{1}(1-s)^{p-2}\left(\log \frac{e}{1-s}\right)^{p / 2} d s \\
& \asymp \frac{1}{(1-|a|)^{p \gamma}}\left(\log \frac{e}{1-|a|}\right)^{p / 2} \quad|a| \rightarrow 1^{-} .
\end{aligned}
$$

Moreover,

$$
\begin{aligned}
I_{1}(|a|) \leq & \left(\log \frac{e}{1-|a|}\right)^{p / 2} \int_{0}^{|a|} \frac{(1-s)^{p-2} d s}{(1-|a| s)^{p \gamma+p-1}} \\
& \leq C\left(\log \frac{e}{1-|a|}\right)^{p / 2} \frac{1}{(1-|a|)^{p \gamma}} .
\end{aligned}
$$

which together with (4.8) and (4.9) gives

$$
\left\|g_{a}\right\|_{B_{p, \log p / 2}} \asymp(1-|a|)^{-\gamma}\left(\log \frac{e}{1-|a|}\right)^{1 / 2}, \quad|a| \rightarrow 1^{-} .
$$

Now, if $1<p \leq 2$, by (4.10), the description of Hilbert-Schmidt integration operators obtained in (1.2), and part (b) of Theorem 3,

$$
\begin{aligned}
& (1-|a|)^{-\gamma}\left(\log \frac{e}{1-|a|}\right)^{1 / 2} \asymp\left\|g_{a}\right\|_{B_{2, \log 1}} \asymp\left\|T_{g_{a}}\right\|_{\mathcal{S}_{2}(\mathcal{D})} \leq\left\|T_{g_{a}}\right\|_{\mathcal{S}_{p}(\mathcal{D})} \\
& \leq C\left\|g_{a}\right\|_{B_{p, \log p / 2}} \asymp(1-|a|)^{-\gamma}\left(\log \frac{e}{1-|a|}\right)^{1 / 2}, \quad|a| \rightarrow 1^{-} .
\end{aligned}
$$

Furthermore, if $2 \leq p<\infty$, using again (4.10) and Proposition 4.3 below,

$$
\begin{aligned}
& (1-|a|)^{-\gamma}\left(\log \frac{e}{1-|a|}\right)^{1 / 2} \asymp\left\|g_{a}\right\|_{B_{p, \log p / 2}} \leq C\left\|T_{g_{a}}\right\|_{\mathcal{S}_{p}(\mathcal{D})} \leq C\left\|T_{g_{a}}\right\|_{\mathcal{S}_{2}(\mathcal{D})} \\
& \asymp\left\|g_{a}\right\|_{B_{2, \log 1}} \asymp(1-|a|)^{-\gamma}\left(\log \frac{e}{1-|a|}\right)^{1 / 2}, \quad|a| \rightarrow 1^{-},
\end{aligned}
$$

and this completes the proof of (b).

Bearing in mind that $\left(X_{\alpha}^{p},\|\cdot\|_{X_{\alpha}^{p}}\right)$ is a Banach space for $p>1$, the closed graph theorem and Lemma 4.1 and Lemma 4.2, we deduce that $X_{0}^{p} \subsetneq B_{p}$ and is different from $B_{p, \log ^{p / 2}}$. In particular, Proposition 3.1 (iv) does not remain true for $\alpha=0$ and $1<p<2$.

4.3. Case $\mathbf{p}>\mathbf{2}$. We collect our results for this range of values of $p$ in the next proposition.

Proposition 4.3. Assume that $g \in H(\mathbb{D})$ and $2 \leq p<\infty$.

(i) If $T_{g} \in \mathcal{S}_{p}(\mathcal{D})$ then $g \in B_{p, \log ^{p / 2}}$.

(ii) If $T_{g} \in \mathcal{S}_{p}(\mathcal{D})$ then $g \in X_{0}^{p}$. 
(iii) Assume that $2<p \leq 4$. If

$$
\|g\|_{X_{0, \log p / 4}^{p}}^{p} \stackrel{\text { def }}{=}|g(0)|^{p}+\int_{\mathbb{D}}\left(\int_{\mathbb{D}} \frac{\left|g^{\prime}(z)\right|^{2}}{|1-\bar{w} z|^{2}} d A(z)\right)^{p / 2}\left(\log \frac{e}{1-|w|}\right)^{p / 4} d A_{p-2}(w)<\infty,
$$

then $T_{g} \in \mathcal{S}_{p}(\mathcal{D})$.

Proof. We prove part (i) first. It is clear that $T_{g} \in \mathcal{S}_{p}(\mathcal{D})$ if and only if $M_{g^{\prime}} \in \mathcal{S}_{p}\left(\mathcal{D}, A^{2}\right)$, which is equivalent to the fact that the adjoint $M_{g^{\prime}}^{*}$ belongs to $\mathcal{S}_{p}\left(A^{2}, \mathcal{D}\right)$. Now, the result can be deduced by applying Proposition 2.1 with $T=M_{g^{\prime}}^{*}$ and $H=\mathcal{D}$. Indeed, an easy computation using (2.6), the properties of the adjoint and the reproducing kernels gives

$$
\begin{aligned}
\left\|M_{g^{\prime}}^{*} B_{z}^{0}\right\|_{\mathcal{D}}^{2} & =\left\langle M_{g^{\prime}}^{*} B_{z}^{0}, M_{g^{\prime}}^{*} B_{z}^{0}\right\rangle_{\mathcal{D}}=\left\langle B_{z}^{0}, M_{g^{\prime}} M_{g^{\prime}}^{*} B_{z}^{0}\right\rangle_{A^{2}}=\overline{M_{g^{\prime}} M_{g^{\prime}}^{*} B_{z}^{0}(z)} \\
& =\overline{g^{\prime}(z) M_{g^{\prime}}^{*} B_{z}^{0}(z)}=\overline{g^{\prime}(z)\left\langle M_{g^{\prime}}^{*} B_{z}^{0}, K_{z}^{\mathcal{D}}\right\rangle_{\mathcal{D}}}=\overline{g^{\prime}(z)\left\langle B_{z}^{0}, M_{g^{\prime}} K_{z}^{\mathcal{D}}\right\rangle_{A^{2}}} \\
& =\overline{g^{\prime}(z)} M_{g^{\prime}} K_{z}^{\mathcal{D}}(z)=\left|g^{\prime}(z)\right|^{2} \log \frac{e}{1-|z|^{2}} .
\end{aligned}
$$

This, bearing in mind (2.4), yields

$$
\int_{\mathbb{D}}\left|g^{\prime}(z)\right|^{p}\left(\log \frac{e}{1-|z|^{2}}\right)^{p / 2}\left(1-|z|^{2}\right)^{p-2} d A(z) \asymp \int_{\mathbb{D}}\left\|M_{g^{\prime}}^{*} b_{z}^{0}\right\|_{\mathcal{D}}^{p} d \lambda(z),
$$

which together with Proposition 2.1, gives (i). Part (ii) follows from Proposition 3.2. Finally, reasoning as in the proof of Theorem 1 (case $2<p \leq 4$ ), we obtain part (iii).

By arguing now similarly as in the proof of Lemma 4.2, we deduce that

$$
\left\|g_{a}\right\|_{X_{0, \log p / 4}^{p}} \asymp(1-|a|)^{-\gamma}\left(\log \frac{1}{1-|a|}\right)^{\frac{1}{4}+\frac{1}{p}}, \quad|a| \rightarrow 1^{-},
$$

and Proposition 4.3, together with Lemma 4.1 and Lemma 4.2, says that any of those conditions which appear in Proposition 4.3 does not describe the membership of $T_{g}$ in $S_{p}(\mathcal{D})$ for $p>2$. However, if the monomials are taken as the symbols, Lemma 4.1 says that the correct behavior of $\left\|T_{g}\right\|_{\mathcal{S}_{p}(\mathcal{D})}$ is given by $X_{0}^{p}$, but if the symbols are the family of functions $g_{a}(z)=(1-\bar{a} z)^{-\gamma}, a \in \mathbb{D}$, Lemma 4.2 says that the correct behavior is given by the $B_{p, \log ^{p / 2}}$ condition.

\section{Relationship With OTHER OPERATORS}

It should be noticed that the integration operator $T_{g}$ is bounded, compact (in $\mathcal{D}_{\alpha}$ ), or belongs to the Schatten class $\mathcal{S}_{p}\left(\mathcal{D}_{\alpha}\right)$ if and only if the multiplication operator $M_{g^{\prime}}: \mathcal{D}_{\alpha} \rightarrow A_{\alpha}^{2}$ is bounded, compact, or belongs to $\mathcal{S}_{p}$. In this section, we shall study the relationship of the integration operator $T_{g}$ (equivalently $M_{g^{\prime}}$ ) with other linear operators such as Toeplitz operators, the big and small Hankel operators, or other multiplication operators. 
5.1. Toeplitz operators. We recall that given a finite positive Borel measure $\mu$ on $\mathbb{D}$, the Toeplitz operator $Q_{\mu}$ on $\mathcal{D}_{\alpha}, \alpha>0$ is defined by

$$
Q_{\mu} f(z)=\int_{\mathbb{D}} f(w) \overline{K_{z}^{\alpha}(w)} d \mu(w), \quad f \in \mathcal{D}_{\alpha}
$$

Toeplitz operators have been a key tool for studying the membership in $\mathcal{S}_{p}$ of many classes of operators, such as composition operators (see [11], [10, Section 7] and [30, Chapter 11]) or integration operators (see $[4,5]$ and $\left[16\right.$, Chapter 6]). Indeed, the integration operator $T_{g}$ and the Toeplitz operator $Q_{\mu}$ on $\mathcal{D}_{\alpha}$ are related via the identity $T_{g}^{*} T_{g}=Q_{\mu_{g}}$, where $\mu_{g}$ is the measure defined by $d \mu_{g}(z)=\left|g^{\prime}(z)\right|^{2} d A_{\alpha}(z)$, and one can obtain a proof of Theorem A using the characterization of Schatten class Toeplitz operators obtained by D. Luecking (see (5.1) below). So, it is natural to expect that the methods used to study the membership of $T_{g}$ in the Schatten $p$-class of $\mathcal{D}_{\alpha}$ are going to work also for the Toeplitz operator $Q_{\mu}$ on $\mathcal{D}_{\alpha}$ for a general measure $\mu$. Before doing that, we recall Luecking's result [10] describing the membership in $\mathcal{S}_{p}\left(\mathcal{D}_{\alpha}\right)$ of the Toeplitz operator $Q_{\mu}$ for all $p>0$ with $p(1-\alpha)<1$. He shows that, for the range of $p$ considered above, $Q_{\mu} \in \mathcal{S}_{p}\left(\mathcal{D}_{\alpha}\right)$ if and only if, for any $r$-lattice $\left\{a_{j}\right\}$ with associated hyperbolic disks $\left\{D_{j}\right\}$

$$
\sum_{j}\left(\frac{\mu\left(D_{j}\right)}{\left(1-\left|a_{j}\right|\right)^{\alpha}}\right)^{p}<\infty
$$

Given a finite positive Borel measure on $\mathbb{D}$, for any $-1<\alpha<\infty$ and $0<p<\infty$ we define

$$
X_{\alpha}^{p}(\mu) \stackrel{\text { def }}{=} \int_{\mathbb{D}}\left(\left(1-|w|^{2}\right)^{\alpha} \int_{\mathbb{D}} \frac{d \mu(z)}{|1-\bar{w} z|^{2+2 \alpha}}\right)^{p / 2}\left(1-|w|^{2}\right)^{p-2} d A(w) .
$$

Here we are able to obtain a full description of the measures $\mu$ for which the Toeplitz operator $Q_{\mu}$ belongs to $\mathcal{S}_{p}\left(\mathcal{D}_{\alpha}\right)$ on the extended range of all $p>0$ with $p(1-\alpha)<2$ and $1<p(2+\alpha)$. We remark here that, as $\alpha>0$, a complete description of the Hilbert-Schmidt Toeplitz operators on $\mathcal{D}_{\alpha}$ is obtained.

Theorem 5.1. Let $\mu$ be a finite positive Borel measure on $\mathbb{D}, \alpha>0$, and let $p>0$ with $1<p(2+\alpha)$ and $p(1-\alpha)<2$. Then the Toeplitz operator $Q_{\mu}$ belongs to $\mathcal{S}_{p}\left(\mathcal{D}_{\alpha}\right)$ if and only if $X_{\alpha}^{2 p}(\mu)<\infty$.

Proof. Consider the inclusion operator $I_{\mu}: \mathcal{D}_{\alpha} \rightarrow L^{2}(\mathbb{D}, \mu)$. It is easy to check that $Q_{\mu}=I_{\mu}^{*} I_{\mu}$, and thus $Q_{\mu} \in \mathcal{S}_{p}\left(\mathcal{D}_{\alpha}\right)$ if and only if $I_{\mu}$ belongs to $\mathcal{S}_{2 p}$. Now, the necessity of $X_{\alpha}^{2 p}(\mu)<\infty$ for $p \geq 1$ and the sufficiency for $p \leq 1$ follow from Proposition 2.2. Also, by repeating the proof of the sufficiency in Theorem 1 replacing the measure $\left|g^{\prime}(z)\right|^{2} d A_{\alpha}(z)$ in that proof 
by the measure $d \mu$ we obtain

$$
\sum_{n}\left\|I_{\mu} e_{n}\right\|_{L^{2}(\mathbb{D}, \mu)}^{2 p} \leq C<\infty
$$

for all orthonormal sets $\left\{e_{n}\right\}$ of $\mathcal{D}_{\alpha}$ provided $p>1$ and $p(1-\alpha)<2$. This proves the sufficiency of $X_{\alpha}^{2 p}(\mu)<\infty$ in that range. Finally, it remains to show the necessity in the case $1 /(2+\alpha)<p<1$. Let $\left\{a_{j}\right\}$ be an $r$-lattice with associated hyperbolic disks $\left\{D_{j}\right\}$. Using that $|1-\bar{w} z| \asymp\left|1-\bar{a}_{j} z\right|$ for $w \in D_{j}$ and Lemma B, we deduce

$$
\begin{aligned}
X_{\alpha}^{2 p}(\mu) & \leq C \int_{\mathbb{D}}\left(\sum_{j} \frac{\mu\left(D_{j}\right)}{\left|1-\bar{a}_{j} z\right|^{2+2 \alpha}}\right)^{p}\left(1-|z|^{2}\right)^{2 p-2+\alpha p} d A(z) \\
& \leq C \sum_{j} \mu\left(D_{j}\right)^{p} \int_{\mathbb{D}} \frac{\left(1-|z|^{2}\right)^{2 p-2+\alpha p}}{\left|1-\bar{a}_{j} z\right|^{2 p+2 \alpha p}} d A(z) \\
& \leq C \sum_{j} \frac{\mu\left(D_{j}\right)^{p}}{\left(1-\left|a_{j}\right|^{2}\right)^{\alpha p}} .
\end{aligned}
$$

Thus, by Luecking's condition (5.1), if $Q_{\mu} \in \mathcal{S}_{p}\left(\mathcal{D}_{\alpha}\right)$ then $X_{\alpha}^{2 p}(\mu)<\infty$ completing the proof of the Theorem.

We conclude this subsection mentioning that in [19] one can find a description of the membership of the Toeplitz operator $Q_{\mu}$ in $\mathcal{S}_{2 k}\left(\mathcal{D}_{\alpha}\right)$ for positive integers $k$ in terms of some iterated integrals.

5.2. Big and small Hankel operators. As in [26] and [20], for $\alpha \geq 0$, we consider the Sobolev space $L_{\alpha}^{2}$ consisting of those differentiable functions $u: \mathbb{D} \rightarrow \mathbb{C}$ for which the norm

$$
\|u\|_{L_{\alpha}^{2}}=\left(|u(0)|^{2}+\int_{\mathbb{D}}|\nabla u(z)|^{2} d A_{\alpha}(z)\right)^{1 / 2}
$$

is finite. It is clear that $\mathcal{D}_{\alpha}$ is a closed subspace of $L_{\alpha}^{2}$. Let $P_{\alpha}$ be the orthogonal projection from $L_{\alpha}^{2}$ onto $\mathcal{D}_{\alpha}$. The big Hankel operator $H_{g}^{\alpha}$ : $\mathcal{D}_{\alpha} \rightarrow L_{\alpha}^{2}$ and the small Hankel operator $h_{g}^{\alpha}: \mathcal{D}_{\alpha} \rightarrow L_{\alpha}^{2}$ are defined by

$$
\begin{aligned}
& H_{g}^{\alpha}(f)=\left(I-P_{\alpha}\right)(\bar{g} f), \\
& h_{g}^{\alpha}(f)=\overline{P_{\alpha}(\bar{f} g)} .
\end{aligned}
$$

The relation between the big Hankel operator and the multiplication operator $M_{g^{\prime}}$ is clear and well understood. Indeed, in [26, Corollary 1] Z. Wu shows that $M_{g^{\prime}}: \mathcal{D}_{\alpha} \rightarrow A_{\alpha}^{2}$ is bounded, compact, or belongs to $\mathcal{S}_{p}$ with $1<p<\infty$, if and only if the same is true for the big Hankel operator $H_{g}^{\alpha}: \mathcal{D}_{\alpha} \rightarrow L_{\alpha}^{2}$. However, although $M_{\bar{g}^{\prime}}$ is related with the the small Hankel operator (see (5.4) below), the transformation of a result from one operator to the other is not straightforward. Respect to this question, it is known that $M_{g^{\prime}}^{\alpha}: \mathcal{D}_{\alpha} \rightarrow A_{\alpha}^{2}$ is bounded (or compact) if and only if $h_{g}^{\alpha}: \mathcal{D}_{\alpha} \rightarrow L_{\alpha}^{2}$ is bounded (or compact) (see Theorem 2 and Lemma 3.3 of [27]). Z. Wu 
also shows that (in the case of the Dirichlet space) for $p \geq 2, H_{g}^{0}: \mathcal{D} \rightarrow L_{0}^{2}$ belongs to $\mathcal{S}_{p}$ if and only if $h_{g}^{0}: \mathcal{D} \rightarrow L_{0}^{2}$ belongs to $\mathcal{S}_{p}$ (see [26, Theorem 6]. Note that, by the previous observations, we may replace $H_{g}^{0}$ by $M_{g^{\prime}}$ or $T_{g}$ ). The main aim of this section consists of extending Wu's result on Schatten $p$-classes for the small Hankel operator to all $\mathcal{D}_{\alpha}$ and to all $p$ with $1<p<\infty$. Before that, we recall that

$$
P_{\alpha} u(w)=u(0)+\int_{\mathbb{D}} \frac{\partial u}{\partial z}(z) \frac{\overline{\partial K_{w}^{\alpha}(z)}}{\partial z} d A_{\alpha}(z),
$$

and has the property (see $[20$, p.105]) that

$$
\frac{\partial}{\partial w}\left(P_{\alpha} u\right)(w)=\int_{\mathbb{D}} \frac{\partial u}{\partial z}(z) \frac{d A_{\alpha}(z)}{(1-\bar{z} w)^{2+\alpha}}, \quad u \in L_{\alpha}^{2} .
$$

Theorem 5.2. Let $\alpha \geq 0, g \in H(\mathbb{D})$ and $1<p<\infty$. Then $T_{g} \in \mathcal{S}_{p}\left(\mathcal{D}_{\alpha}\right)$ if and only if $h_{g}^{\alpha} \in \mathcal{S}_{p}\left(\mathcal{D}_{\alpha}, L_{\alpha}^{2}\right)$.

Proof. Firstly, we recall that if $T_{g}$ or $h_{g}^{\alpha}$ is bounded, then $g \in \mathcal{D}_{\alpha}$. It is enough to consider the relationship between $M_{\bar{g}^{\prime}}$ and $h_{g}^{\alpha}$. For this, we look at the difference of $M_{\overline{g^{\prime}}}$ and $\frac{\partial}{\partial \bar{w}} h_{g}^{\alpha}$. For $f \in \mathcal{D}_{\alpha}$, a straightforward calculation using that $g \in \mathcal{D}_{\alpha}$ and (5.3) yields

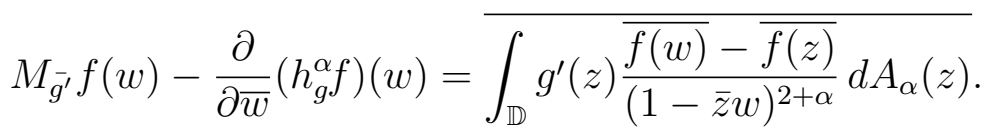

For $1<p<\infty$, if $T_{g} \in \mathcal{S}_{p}\left(\mathcal{D}_{\alpha}\right)$ or $h_{g}^{\alpha} \in \mathcal{S}_{p}\left(\mathcal{D}_{\alpha}, L_{\alpha}^{2}\right)$ then $g \in B_{p}$ (see Propositions 3.1, 3.2, 3.3, Theorem 3 and [26, Theorem 1]), and therefore the difference considered above, as an operator acting from $\mathcal{D}_{\alpha}$ into $L^{2}\left(\mathbb{D}, d A_{\alpha}\right)$, belongs to $\mathcal{S}_{p}$, by Proposition 5.3 (which we are going to prove below). This completes the proof.

For $u \in L^{2}\left(\mathbb{D}, d A_{\alpha}\right)$, consider the operator

$$
\Delta_{u} f(w)=\int_{\mathbb{D}} u(z) \frac{\overline{f(w)}-\overline{f(z)}}{(1-\bar{z} w)^{2+\alpha}} d A_{\alpha}(z) .
$$

Proposition 5.3. Let $\alpha \geq 0, u \in A_{\alpha}^{2}$ and $1<p<\infty$. If $u \in A_{p-2}^{p}$, then $\Delta_{u}: \mathcal{D}_{\alpha} \rightarrow L^{2}\left(\mathbb{D}, d A_{\alpha}\right)$ belongs to $\mathcal{S}_{p}$.

For the proof of that proposition, we need the following lemma.

Lemma 5.4. Let $\sigma>-1$, and $2+\sigma<b \leq 4+2 \sigma$. Then for each $a \in \mathbb{D}$ and any $f \in H(\mathbb{D})$ we have

$$
\int_{\mathbb{D}} \frac{|f(z)-f(a)|^{2}}{|1-\bar{a} z|^{b}} d A_{\sigma}(z) \leq C \int_{\mathbb{D}}\left|f^{\prime}(z)\right|^{2} \frac{d A_{2+\sigma}(z)}{|1-\bar{a} z|^{b}} .
$$

Proof. Let $\varphi_{a}(z)=\frac{a-z}{1-\bar{a} z}$, and consider the function $f_{a}=\left(f \circ \varphi_{a}\right)$. After the change of variables $z=\varphi_{a}(\zeta)$, and an application of Lemma 2.1 of [7] we 
get

$$
\begin{aligned}
\int_{\mathbb{D}} \frac{|f(z)-f(a)|^{2}}{|1-\bar{a} z|^{b}} d A_{\sigma}(z) & =\left(1-|a|^{2}\right)^{2+\sigma-b} \int_{\mathbb{D}} \frac{\left|f_{a}(\zeta)-f_{a}(0)\right|^{2}}{|1-\bar{a} \zeta|^{4+2 \sigma-b}} d A_{\sigma}(\zeta) \\
& \leq C\left(1-|a|^{2}\right)^{2+\sigma-b} \int_{\mathbb{D}} \frac{\left|\left(f_{a}\right)^{\prime}(\zeta)\right|^{2} d A_{2+\sigma}(\zeta)}{|1-\bar{a} \zeta|^{4+2 \sigma-b}}
\end{aligned}
$$

Finally, the change of variables $\zeta=\varphi_{a}(z)$ gives

$$
\begin{aligned}
\int_{\mathbb{D}} \frac{|f(z)-f(a)|^{2}}{|1-\bar{a} z|^{b}} d A_{\sigma}(z) & \leq C \int_{\mathbb{D}}\left|f^{\prime}(z)\right|^{2} \frac{|1-\bar{a} z|^{4+2 \sigma-b}}{\left(1-|a|^{2}\right)^{2+\sigma}}\left(1-\left|\varphi_{a}(z)\right|^{2}\right)^{2+\sigma} d A(z) \\
& =C \int_{\mathbb{D}}\left|f^{\prime}(z)\right|^{2} \frac{\left(1-|z|^{2}\right)^{2+\sigma}}{|1-\bar{a} z|^{b}} d A(z) .
\end{aligned}
$$

Proof of Proposition 5.3. Firstly we deal with the case $p \geq 2$. Note that, for $f \in H^{\infty}$ (the algebra of all bounded analytic functions on $\mathbb{D}$, a dense subset of $\left.\mathcal{D}_{\alpha}\right)$ and $u$ analytic, one has $\Delta_{u} f=u \bar{f}-\widetilde{P_{\alpha}}(u \bar{f})$, where $\widetilde{P_{\alpha}}$ denotes the Bergman projection from $L^{2}\left(\mathbb{D}, d A_{\alpha}\right)$ to $A_{\alpha}^{2}$. Therefore, $\Delta_{u} f$ is the solution of the equation $\bar{\partial} v=u \overline{f^{\prime}}$ with minimal $L^{2}\left(\mathbb{D}, d A_{\alpha}\right)$ norm. Now, it is well known that the solution of $\bar{\partial} v=u \overline{f^{\prime}}$ given by

$$
v(z)=\int_{\mathbb{D}} \frac{\left(u \overline{f^{\prime}}\right)(w)\left(1-|w|^{2}\right)^{1+\alpha}}{(z-w)(1-\bar{w} z)^{1+\alpha}} d A(w)
$$

satisfies the estimate

$$
\int_{\mathbb{D}}|v(z)|^{2} d A_{\alpha}(z) \leq C \int_{\mathbb{D}}\left|\left(u \overline{f^{\prime}}\right)(z)\right|^{2}\left(1-|z|^{2}\right)^{2+\alpha} d A(z)
$$

Indeed, the estimate in question follows from Cauchy-Schwarz inequality and the fact that, for $c>0$ and $t>-1$, the integral $\int_{\mathbb{D}} \frac{\left(1-|w|^{2}\right)^{t} d A(w)}{|z-w||1-\bar{w} z|^{1+t+c}}$ is comparable to $\left(1-|z|^{2}\right)^{-c}$ (this is just a variant of Lemma B). Taking all of this into account, we obtain that

$$
\left\|\Delta_{u} f\right\|_{L^{2}\left(\mathbb{D}, d A_{\alpha}\right)}^{2} \leq C \int_{\mathbb{D}}\left|u(z) f^{\prime}(z)\right|^{2}\left(1-|z|^{2}\right)^{2+\alpha} d A(z)
$$

From this inequality, it follows easily that the operator $\Delta_{u}$ is bounded (or compact) if $\sup _{z \in \mathbb{D}}(1-|z|)|u(z)|<\infty$ (or if $\left.\lim _{|z| \rightarrow 1^{-}}(1-|z|)|u(z)|=0\right)$, and it is clear that these conditions are implied by the fact that $u \in A_{p-2}^{p}$. Now, let $\left\{e_{n}\right\}$ be any orthonormal set in $\mathcal{D}_{\alpha}$. Therefore, using (5.5), Hölder's 
inequality, (2.3) and (2.7), we obtain

$$
\begin{aligned}
\sum_{n}\left\|\Delta_{u} e_{n}\right\|_{L^{2}\left(\mathbb{D}, d A_{\alpha}\right)}^{p} & \leq C \sum_{n}\left(\int_{\mathbb{D}}\left|u(z) e_{n}^{\prime}(z)\right|^{2}\left(1-|z|^{2}\right)^{2+\alpha} d A(z)\right)^{p / 2} \\
& \leq C \sum_{n} \int_{\mathbb{D}}|u(z)|^{p}\left|e_{n}^{\prime}(z)\right|^{2}\left(1-|z|^{2}\right)^{p+\alpha} d A(z) \\
& =C \int_{\mathbb{D}}|u(z)|^{p} \sum_{n}\left|e_{n}^{\prime}(z)\right|^{2}\left(1-|z|^{2}\right)^{p+\alpha} d A(z) \\
& \leq C\|u\|_{A_{p-2}^{p}}^{p} .
\end{aligned}
$$

A different proof for the case $p=2$ (that can be adapted to the case $p>2$ ) can be given as follows. Let $\left\{e_{n}\right\}$ be any orthonormal basis of $\mathcal{D}_{\alpha}$. Take $0<\varepsilon<1$. Then, Lemma 5.4 yields

$$
\begin{aligned}
\left|\Delta_{u} e_{n}(w)\right|^{2} & \leq\left(\int_{\mathbb{D}} \frac{|u(z)|^{2} d A_{\alpha+\varepsilon}(z)}{|1-\bar{w} z|^{2+\alpha}}\right)\left(\int_{\mathbb{D}} \frac{\left|e_{n}(w)-e_{n}(z)\right|^{2} d A_{\alpha-\varepsilon}(z)}{|1-\bar{w} z|^{2+\alpha}}\right) \\
& \leq C\left(\int_{\mathbb{D}} \frac{|u(z)|^{2} d A_{\alpha+\varepsilon}(z)}{|1-\bar{w} z|^{2+\alpha}}\right)\left(\int_{\mathbb{D}} \frac{\left|e_{n}^{\prime}(z)\right|^{2} d A_{2+\alpha-\varepsilon}(z)}{|1-\bar{w} z|^{2+\alpha}}\right) .
\end{aligned}
$$

Therefore, using (2.3) and Lemma B, we get

$$
\begin{aligned}
\sum_{n} & \left\|\Delta_{u} e_{n}\right\|^{2}=\sum_{n} \int_{\mathbb{D}}\left|\Delta_{u} e_{n}(w)\right|^{2} d A_{\alpha}(w) \\
& \leq C \int_{\mathbb{D}}\left(\int_{\mathbb{D}} \frac{|u(z)|^{2} d A_{\alpha+\varepsilon}(z)}{|1-\bar{w} z|^{2+\alpha}}\right)\left(\int_{\mathbb{D}} \frac{\sum_{n}\left|e_{n}^{\prime}(z)\right|^{2} d A_{2+\alpha-\varepsilon}(z)}{|1-\bar{w} z|^{2+\alpha}}\right) d A_{\alpha}(w) \\
& \leq C \int_{\mathbb{D}}\left(\int_{\mathbb{D}} \frac{|u(z)|^{2} d A_{\alpha+\varepsilon}(z)}{|1-\bar{w} z|^{2+\alpha}}\right)\left(\int_{\mathbb{D}} \frac{d A_{-\varepsilon}(z)}{|1-\bar{w} z|^{2+\alpha}}\right) d A_{\alpha}(w) \\
& \leq C \int_{\mathbb{D}}\left(\int_{\mathbb{D}} \frac{|u(z)|^{2} d A_{\alpha+\varepsilon}(z)}{|1-\bar{w} z|^{2+\alpha}}\right) d A_{-\varepsilon}(w) \\
& =C \int_{\mathbb{D}}|u(z)|^{2}\left(\int_{\mathbb{D}} \frac{d A_{-\varepsilon}(w)}{|1-\bar{w} z|^{2+\alpha}}\right) d A_{\alpha+\varepsilon}(z) \leq C\|u\|_{A^{2}}^{2} .
\end{aligned}
$$

For $1<p<2$, one has $A_{p-2}^{p} \subset A^{2}$. Thus, by the case we have just proved, the operator $\Delta_{u}$ is Hilbert-Schmidt and, in particular, compact. By Proposition 2.2, a sufficient condition for $\Delta_{u}$ to be in the class $\mathcal{S}_{p}$ is

$$
\int_{\mathbb{D}}\left\|\Delta_{u} j_{z}^{\alpha}\right\|_{L^{2}\left(\mathbb{D}, d A_{\alpha}\right)}^{p} d \lambda(z)<\infty
$$


Now, take $0<\varepsilon<1$ with $\alpha-\varepsilon>-1$ and $p-\varepsilon p>1$. Proceeding as in (5.6), and then using Lemma $\mathrm{C}$ we obtain

$$
\begin{aligned}
\left|\left(\Delta_{u} J_{z}^{\alpha}\right)(w)\right|^{2} & \leq C\left(\int_{\mathbb{D}} \frac{|u(\zeta)|^{2} d A_{\alpha+\varepsilon}(\zeta)}{|1-\bar{w} \zeta|^{2+\alpha-\varepsilon}}\right)\left(\int_{\mathbb{D}} \frac{\left|\left(J_{z}^{\alpha}\right)^{\prime}(\zeta)\right|^{2} d A_{2+\alpha-\varepsilon}(\zeta)}{|1-\bar{w} \zeta|^{2+\alpha+\varepsilon}}\right) \\
& \leq C\left(\int_{\mathbb{D}} \frac{|u(\zeta)|^{2} d A_{\alpha+\varepsilon}(\zeta)}{|1-\bar{w} \zeta|^{2+\alpha-\varepsilon}}\right)\left(\int_{\mathbb{D}} \frac{d A_{2+\alpha-\varepsilon}(\zeta)}{|1-\bar{z} \zeta|^{4+2 \alpha}|1-\bar{w} \zeta|^{2+\alpha+\varepsilon}}\right) \\
& \leq C\left(\int_{\mathbb{D}} \frac{|u(\zeta)|^{2} d A_{\alpha+\varepsilon}(\zeta)}{|1-\bar{w} \zeta|^{2+\alpha-\varepsilon}}\right) \frac{\left(1-|z|^{2}\right)^{-\alpha-\varepsilon}}{|1-\bar{w} z|^{2+\alpha+\varepsilon}} .
\end{aligned}
$$

This, together with Lemma C, gives

$$
\begin{aligned}
& \left\|\Delta_{u} J_{z}^{\alpha}\right\|_{L^{2}\left(\mathbb{D}, d A_{\alpha}\right)}^{2}=\int_{\mathbb{D}}\left|\left(\Delta_{u} J_{z}^{\alpha}\right)(w)\right|^{2} d A_{\alpha}(w) \\
& \quad \leq C\left(1-|z|^{2}\right)^{-\alpha-\varepsilon} \int_{\mathbb{D}}|u(\zeta)|^{2}\left(\int_{\mathbb{D}} \frac{d A_{\alpha}(w)}{|1-\bar{w} \zeta|^{2+\alpha-\varepsilon}|1-\bar{w} z|^{2+\alpha+\varepsilon}}\right) d A_{\alpha+\varepsilon}(\zeta) \\
& \quad \leq C\left(1-|z|^{2}\right)^{-\alpha-2 \varepsilon} \int_{\mathbb{D}} \frac{|u(\zeta)|^{2} d A_{\alpha+\varepsilon}(\zeta)}{|1-\bar{z} \zeta|^{2+\alpha-\varepsilon}} .
\end{aligned}
$$

Thus,

$$
\begin{gathered}
\int_{\mathbb{D}}\left\|\Delta_{u} j_{z}^{\alpha}\right\|_{L^{2}\left(\mathbb{D}, d A_{\alpha}\right)}^{p} d \lambda(z)=\int_{\mathbb{D}}\left\|\Delta_{u} J_{z}^{\alpha}\right\|_{L^{2}\left(\mathbb{D}, d A_{\alpha}\right)}^{p}\left\|J_{z}^{\alpha}\right\|_{\mathcal{D}_{\alpha}}^{-p} d \lambda(z) \\
=\int_{\mathbb{D}}\left\|\Delta_{u} J_{z}^{\alpha}\right\|_{L^{2}\left(\mathbb{D}, d A_{\alpha}\right)}^{p}\left(1-|z|^{2}\right)^{(2+\alpha) \frac{p}{2}-2} d A(z) \\
\leq C \int_{\mathbb{D}}\left(\int_{\mathbb{D}} \frac{|u(\zeta)|^{2} d A_{\alpha+\varepsilon}(\zeta)}{|1-\bar{z} \zeta|^{2+\alpha-\varepsilon}}\right)^{\frac{p}{2}}\left(1-|z|^{2}\right)^{p-2-\varepsilon p} d A(z) .
\end{gathered}
$$

Now, consider an $r$-lattice $\left\{a_{n}\right\}$ with associated hyperbolic disks $\left\{D_{n}\right\}$. Since $p / 2 \leq 1$ we have

$$
\begin{aligned}
\left(\int_{\mathbb{D}} \frac{|u(\zeta)|^{2} d A_{\alpha+\varepsilon}(\zeta)}{|1-\bar{z} \zeta|^{2+\alpha-\varepsilon}}\right)^{\frac{p}{2}} & \leq\left(\sum_{n} \int_{D_{n}} \frac{|u(\zeta)|^{2} d A_{\alpha+\varepsilon}(\zeta)}{|1-\bar{z} \zeta|^{2+\alpha-\varepsilon}}\right)^{\frac{p}{2}} \\
& \leq C\left(\sum_{n} \frac{\left(1-\left|a_{n}\right|^{2}\right)^{\alpha+\varepsilon}}{\left|1-\bar{z} a_{n}\right|^{2+\alpha-\varepsilon}} \int_{D_{n}}|u(\zeta)|^{2} d A(\zeta)\right)^{\frac{p}{2}} \\
& \leq C \sum_{n} \frac{\left(1-\left|a_{n}\right|^{2}\right)^{(\alpha+\varepsilon) \frac{p}{2}}}{\left|1-\bar{z} a_{n}\right|^{p+(\alpha-\varepsilon) \frac{p}{2}}}\left(\int_{D_{n}}|u(\zeta)|^{2} d A(\zeta)\right)^{\frac{p}{2}}
\end{aligned}
$$


Putting this into (5.8) and applying Lemma B, we obtain

$$
\begin{aligned}
\int_{\mathbb{D}}\left\|\Delta_{u} j_{z}^{\alpha}\right\|_{L^{2}\left(\mathbb{D}, d A_{\alpha}\right)}^{p} d \lambda(z) & \\
\leq & \leq \sum_{n}\left(1-\left|a_{n}\right|^{2}\right)^{(\alpha+\varepsilon) \frac{p}{2}}\left(\int_{D_{n}}|u(\zeta)|^{2} d A(\zeta)\right)^{\frac{p}{2}} \int_{\mathbb{D}} \frac{\left(1-|z|^{2}\right)^{p-2-\varepsilon p} d A(z)}{\left|1-\bar{z} a_{n}\right|^{p+(\alpha-\varepsilon) \frac{p}{2}}} \\
& \leq C \sum_{n}\left(\int_{D_{n}}|u(\zeta)|^{2} d A(\zeta)\right)^{\frac{p}{2}} \leq C\|u\|_{A_{p-2}^{p}}^{p}
\end{aligned}
$$

due to Theorem 0 of [5]. This establishes (5.7) completing the proof.

5.3. Multiplication operators. It is well known that the multiplication operator $M_{g^{\prime}}: \mathcal{D}_{\alpha} \rightarrow A_{\alpha}^{2}$ is bounded or compact if and only if $M_{g^{\prime \prime}}: \mathcal{D}_{\alpha} \rightarrow$ $A_{2+\alpha}^{2}$ is bounded or compact. Thus, a natural question arises here: It is true that $M_{g^{\prime}}: \mathcal{D}_{\alpha} \rightarrow A_{\alpha}^{2}$ is in the Schatten class $\mathcal{S}_{p}$ if and only if $M_{g^{\prime \prime}}: \mathcal{D}_{\alpha} \rightarrow A_{2+\alpha}^{2}$ belongs to $S_{p}$ ? We are going to see that this happens when $p>1$, but the result is false for $p=1$. Let us consider the spaces $\dot{A}_{\alpha}^{2}=\left\{f \in A_{\alpha}^{2}: f(0)=0\right\}$ and $\dot{\mathcal{D}}_{\alpha}=\left\{f \in \mathcal{D}_{\alpha}: f(0)=0\right\}$.

Theorem 5.5. Let $\alpha \geq 0,1<p<\infty$ and $g \in H(\mathbb{D})$. The following are equivalent:

(a) $M_{g^{\prime}}: \dot{\mathcal{D}}_{\alpha} \rightarrow \dot{A}_{\alpha}^{2}$ is in $\mathcal{S}_{p}$

(b) $M_{g^{\prime \prime}}: \dot{\mathcal{D}}_{\alpha} \rightarrow A_{2+\alpha}^{2}$ is in $\mathcal{S}_{p}$.

Taking into account Theorems A and 2, the next result shows that it is no longer true that $M_{g^{\prime}}$ being in the trace class $\mathcal{S}_{1}$ is equivalent to $M_{g^{\prime \prime}}$ being in the trace class. We recall that $g \in B_{1}$ if $g \in H(\mathbb{D})$ and

$$
\int_{\mathbb{D}}\left|g^{\prime \prime}(z)\right| d A(z)<\infty
$$

TheOREM 5.6. Let $g \in H(\mathbb{D})$. Then,

(a) For $\alpha>0, M_{g^{\prime \prime}} \in \mathcal{S}_{1}\left(\mathcal{D}_{\alpha}, A_{2+\alpha}^{2}\right)$ if and only if $g \in B_{1}$.

(b) If $M_{g^{\prime \prime}} \in \mathcal{S}_{1}\left(\mathcal{D}, A_{2}^{2}\right)$ then $g \in B_{1}$.

(c) If

$$
\int_{\mathbb{D}}\left|g^{\prime \prime}(z)\right|\left(\log \frac{e}{1-|z|^{2}}\right)^{1 / 2} d A(z)<\infty,
$$

then $M_{g^{\prime \prime}} \in \mathcal{S}_{1}\left(\mathcal{D}, A_{2}^{2}\right)$.

(d) Neither of the two previous implications (b) and (c) can be reversed. Moreover, there is a function $g \in H(\mathbb{D})$ with $M_{g^{\prime \prime}} \in \mathcal{S}_{1}\left(\mathcal{D}, A_{2}^{2}\right)$ such that

$$
\int_{\mathbb{D}}\left|g^{\prime \prime}(z)\right| \varphi(z) d A(z)=\infty
$$

for any function $\varphi(r)$ increasing continuously to $\infty$ on $(0,1)$. 
One should compare Theorem 5.6 with the results obtained in Theorem 8 of [6], where trace class bilinear Hankel forms on the Dirichlet space are studied.

Proof of Theorem 5.5. We recall that if (a) or (b) holds, then $g \in B_{p}$. We first deal with the case $p \geq 2$. Since $\|f\|_{A_{\alpha}^{2}} \asymp\left\|f^{\prime}\right\|_{A_{2+\alpha}^{2}}$ for $f \in \dot{A}_{\alpha}^{2}$, then, for any orthonormal set $\left\{e_{n}\right\}$ of $\dot{\mathcal{D}}_{\alpha}$, we have

$$
\sum_{n}\left\|M_{g^{\prime}} e_{n}\right\|_{A_{\alpha}^{2}}^{p} \asymp \sum_{n}\left\|\left(M_{g^{\prime}} e_{n}\right)^{\prime}\right\|_{A_{2+\alpha}^{2}}^{p} .
$$

Note that

$$
\begin{aligned}
& \sum_{n}\left\|\left(M_{g^{\prime}} e_{n}\right)^{\prime}\right\|_{A_{2+\alpha}^{2}}^{p} \leq C\left(\sum_{n}\left\|M_{g^{\prime \prime}} e_{n}\right\|_{A_{2+\alpha}^{2}}^{p}+\sum_{n}\left\|M_{g^{\prime}} e_{n}^{\prime}\right\|_{A_{2+\alpha}^{2}}^{p}\right), \\
& \text { and } \\
& \sum_{n}\left\|M_{g^{\prime \prime}} e_{n}\right\|_{A_{2+\alpha}^{2}}^{p} \leq C\left(\sum_{n}\left\|\left(M_{g^{\prime}} e_{n}\right)^{\prime}\right\|_{A_{2+\alpha}^{2}}^{p}+\sum_{n}\left\|M_{g^{\prime}} e_{n}^{\prime}\right\|_{A_{2+\alpha}^{2}}^{p}\right) .
\end{aligned}
$$

Since $g \in B_{p}$, it follows from Hölder's inequality that

$$
\begin{aligned}
\sum_{n}\left\|M_{g^{\prime}} e_{n}^{\prime}\right\|_{A_{2+\alpha}^{2}}^{p} & =\sum_{n}\left(\int_{\mathbb{D}}\left|g^{\prime}(z)\right|^{2}\left|e_{n}^{\prime}(z)\right|^{2} d A_{2+\alpha}(z)\right)^{p / 2} \\
& \leq C \sum_{n} \int_{\mathbb{D}}\left|g^{\prime}(z)\right|^{p}\left|e_{n}^{\prime}(z)\right|^{2}\left(1-|z|^{2}\right)^{p+\alpha} d A(z) \\
& \leq C \int_{\mathbb{D}}\left|g^{\prime}(z)\right|^{p}\left\|J_{z}^{\alpha}\right\|_{\mathcal{D}_{\alpha}^{2}}^{2}\left(1-|z|^{2}\right)^{p+\alpha} d A(z) \\
& =C \int_{\mathbb{D}}\left|g^{\prime}(z)\right|^{p}\left(1-|z|^{2}\right)^{p-2} d A(z) \leq C\|g\|_{B_{p}}^{p}
\end{aligned}
$$

From this, (5.9) and (5.10), it is easy to see that (a) and (b) are equivalent.

Now we deal with the case $1<p<2$. Since $\dot{A}_{\alpha}^{2}$ coincides with $\dot{\mathcal{D}}_{2+\alpha}$ with equivalent norms, we will see that (b) is equivalent to $M_{g^{\prime}}: \dot{\mathcal{D}}_{\alpha} \rightarrow \dot{\mathcal{D}}_{2+\alpha}$ being in $\mathcal{S}_{p}$. For all orthonormal sets $\left\{e_{n}\right\}$ of $\dot{\mathcal{D}}_{\alpha}$ and $\left\{f_{n}\right\}$ of $\dot{\mathcal{D}}_{2+\alpha}$, we have that

$$
\sum_{n}\left|\left\langle M_{g^{\prime}} e_{n}, f_{n}\right\rangle_{\mathcal{D}_{2+\alpha}}\right|^{p} \asymp(I I)
$$

where

$$
(I I)=\sum_{n}\left|\left\langle\left(M_{g^{\prime}} e_{n}\right)^{\prime}, f_{n}^{\prime}\right\rangle_{A_{2+\alpha}^{2}}\right|^{p} .
$$

We see that $(I I) \leq C((I I a)+(I I b))$, where

$$
(I I a)=\sum_{n}\left|\int_{\mathbb{D}} g^{\prime}(z) e_{n}^{\prime}(z) \overline{f_{n}^{\prime}(z)}\left(1-|z|^{2}\right)^{2} d A_{\alpha}(z)\right|^{p},
$$


and

$$
(I I b)=\sum_{n}\left|\int_{\mathbb{D}} g^{\prime \prime}(z) e_{n}(z) \overline{f_{n}^{\prime}(z)}\left(1-|z|^{2}\right)^{2} d A_{\alpha}(z)\right|^{p}
$$

Next, we are going to see that the term $(I I a)$ is dominated by the $B_{p}$ norm of $g$. Indeed, Hölder's inequality gives

$$
\begin{aligned}
(I I a) & \leq \sum_{n}\left(\int_{\mathbb{D}}\left|g^{\prime}(z)\right|\left|e_{n}^{\prime}(z)\right|\left|f_{n}^{\prime}(z)\right|\left(1-|z|^{2}\right)^{2} d A_{\alpha}(z)\right)^{p} \\
& \leq\left(\sum_{n} \int_{\mathbb{D}}\left|g^{\prime}(z)\right|^{p}\left|e_{n}^{\prime}(z)\right|^{p}\left|f_{n}^{\prime}(z)\right|^{2-p}\left(1-|z|^{2}\right)^{2} d A_{\alpha}(z)\right)\left\|f_{n}\right\|_{\mathcal{D}_{2+\alpha}}^{p-1} \\
& \leq \int_{\mathbb{D}}\left|g^{\prime}(z)\right|^{p}\left(\sum_{n}\left|e_{n}^{\prime}(z)\right|^{p}\left|f_{n}^{\prime}(z)\right|^{2-p}\right)\left(1-|z|^{2}\right)^{2} d A_{\alpha}(z) .
\end{aligned}
$$

At this point, we use Hölder's inequality again together with (2.3) and (2.7) to obtain that

$$
\begin{aligned}
(I I a) & \leq \int_{\mathbb{D}}\left|g^{\prime}(z)\right|^{p}\left(\sum_{n}\left|e_{n}^{\prime}(z)\right|^{2}\right)^{p / 2}\left(\sum_{n}\left|f_{n}^{\prime}(z)\right|^{2}\right)^{\frac{2-p}{2}}\left(1-|z|^{2}\right)^{2} d A_{\alpha}(z) \\
& \leq \int_{\mathbb{D}}\left|g^{\prime}(z)\right|^{p}\left\|J_{z}^{\alpha}\right\|_{\mathcal{D}_{\alpha}}^{p}\left\|J_{z}^{2+\alpha}\right\|_{\mathcal{D}_{2+\alpha}}^{2-p}\left(1-|z|^{2}\right)^{2} d A_{\alpha}(z) \\
& =\int_{\mathbb{D}}\left|g^{\prime}(z)\right|^{p}\left(1-|z|^{2}\right)^{-(2+\alpha) \frac{p}{2}}\left(1-|z|^{2}\right)^{-(4+\alpha) \frac{2-p}{2}}\left(1-|z|^{2}\right)^{2} d A_{\alpha}(z) \\
& \leq C\|g\|_{B_{p}}^{p} .
\end{aligned}
$$

Thus, putting all together, we see that if $g \in B_{p}$ then

$$
\sum_{n}\left|\left\langle M_{g^{\prime}} e_{n}, f_{n}\right\rangle_{\mathcal{D}_{2+\alpha}}\right|^{p}<\infty \Leftrightarrow \sum_{n}\left|\left\langle M_{g^{\prime \prime}} e_{n}, f_{n}^{\prime}\right\rangle_{A_{2+\alpha}^{2}}\right|^{p}<\infty
$$

Observe that we have just proved one implication, but the other is proved exactly in the same way. Finally, it is clear that $\left\{f_{n}\right\}$ is an orthonormal set of $\dot{\mathcal{D}}_{2+\alpha}$ if and only if $\left\{f_{n}^{\prime}\right\}$ is an orthonormal set of $A_{2+\alpha}^{2}$, which gives $(a) \Leftrightarrow(b)$.

Proof of Theorem 5.6. If the decomposition of the positive operator $M_{g^{\prime \prime}}^{*} M_{g^{\prime \prime}}$ is given by $\sum_{n} \lambda_{n}\left\langle\cdot, e_{n}\right\rangle_{\mathcal{D}_{\alpha}} e_{n}$, then, as in the proof of Proposition 3.3, $\left\{e_{n}\right\}$ is an orthonormal basis of $\mathcal{D}_{\alpha}$. Thus, by Lemma 2.3, we have that

$$
\left(1-|z|^{2}\right)^{-1-\alpha} \leq C \sum_{n}\left|e_{n}(z)\right|\left|e_{n}^{\prime}(z)\right|
$$


and we deduce

$$
\begin{aligned}
\int_{\mathbb{D}}\left|g^{\prime \prime}(z)\right| d A(z) & \leq C \int_{\mathbb{D}}\left|g^{\prime \prime}(z)\right|\left(1-|z|^{2}\right)\left(\sum_{n}\left|e_{n}(z)\right|\left|e_{n}^{\prime}(z)\right|\right) d A_{\alpha}(z) \\
& =C \sum_{n} \int_{\mathbb{D}}\left|g^{\prime \prime}(z)\right|\left(1-|z|^{2}\right)\left|e_{n}(z)\right|\left|e_{n}^{\prime}(z)\right| d A_{\alpha}(z) \\
& \leq C \sum_{n}\left(\int_{\mathbb{D}}\left|g^{\prime \prime}(z)\right|^{2}\left|e_{n}(z)\right|^{2}\left(1-|z|^{2}\right)^{2} d A_{\alpha}(z)\right)^{1 / 2} \\
& =C \sum_{n}\left\|M_{g^{\prime \prime}} e_{n}\right\|_{A_{2+\alpha}^{2}}=C \sum_{n}\left(\left\langle M_{g^{\prime \prime}} e_{n}, M_{g^{\prime \prime}} e_{n}\right\rangle_{A_{2+\alpha}^{2}}\right)^{1 / 2} \\
& =C \sum_{n}\left|\lambda_{n}\right|^{1 / 2}=C\left\|M_{g^{\prime \prime}}\right\|_{\mathcal{S}_{1}},
\end{aligned}
$$

which gives (b) and the necessity in (a).

Now we proceed to show part (c), and the sufficiency in (a). Bearing in mind (2.3) and (2.4), for all orthonormal sets $\left\{e_{n}\right\}$ of $\mathcal{D}_{\alpha}$ and $\left\{f_{n}\right\}$ of $A_{2+\alpha}^{2}$, we have

$$
\begin{aligned}
\left\|M_{g^{\prime \prime}}\right\|_{\mathcal{S}_{1}} & \leq \sum_{n}\left|\left\langle M_{g^{\prime \prime}} e_{n}, f_{n}\right\rangle_{A_{2+\alpha}^{2}}\right| \\
& \leq \int_{\mathbb{D}}\left|g^{\prime \prime}(z)\right|\left(\sum_{n}\left|e_{n}(z)\right|\left|f_{n}(z)\right|\right) d A_{2+\alpha}(z) \\
& \leq \int_{\mathbb{D}}\left|g^{\prime \prime}(z)\right|\left(\sum_{n}\left|e_{n}(z)\right|^{2}\right)^{1 / 2}\left(\sum_{n}\left|f_{n}(z)\right|^{2}\right)^{1 / 2} d A_{2+\alpha}(z) \\
& \leq \int_{\mathbb{D}}\left|g^{\prime \prime}(z)\right|\left\|K_{z}\right\|_{\mathcal{D}_{\alpha}}\left\|B_{z}^{2+\alpha}\right\|_{A_{2+\alpha}^{2}} d A_{2+\alpha}(z) \\
& \asymp \int_{\mathbb{D}}\left|g^{\prime \prime}(z)\right|\left\|K_{z}\right\|_{\mathcal{D}_{\alpha}} d A_{\alpha / 2}(z)
\end{aligned}
$$

and, according to (2.6), this is comparable to

$$
\int_{\mathbb{D}}\left|g^{\prime \prime}(z)\right|\left(\log \frac{e}{1-|z|^{2}}\right)^{1 / 2} d A(z) \quad \text { if } \quad \alpha=0
$$

establishing part (c); and is comparable to

$$
\int_{\mathbb{D}}\left|g^{\prime \prime}(z)\right| d A(z) \quad \text { for } \quad \alpha>0
$$

that gives the remaining part in (a).

Now we prove (d). To see that part (b) can not be reversed, consider the functions $g_{a}(z)=(1-\bar{a} z)^{-\gamma}, \gamma>0$ and $a \in \mathbb{D}$. Then, the same argument leading to (4.10) yields

$$
\int_{\mathbb{D}}\left|g_{a}^{\prime \prime}(z)\right|\left(\log \frac{e}{1-|z|^{2}}\right)^{1 / 2} d A(z) \asymp\left(1-|a|^{2}\right)^{-\gamma}\left(\log \frac{e}{1-|a|^{2}}\right)^{1 / 2}
$$


and by what we have just proved (see (5.11) and the comments after that), one gets

$$
\left\|M_{g_{a}^{\prime \prime}}\right\|_{\mathcal{S}_{1}\left(\mathcal{D}, A_{2}^{2}\right)} \leq C\left(1-|a|^{2}\right)^{-\gamma}\left(\log \frac{e}{1-|a|^{2}}\right)^{1 / 2} .
$$

On the other hand, we can estimate the trace of $M_{g_{a}^{\prime \prime}}$ from below as follows. Let $\left\{e_{n}\right\}$ be any orthonormal basis of $\mathcal{D}$. Then

$$
\begin{aligned}
\left\|M_{g_{a}^{\prime \prime}}\right\|_{\mathcal{S}_{1}\left(\mathcal{D}, A_{2}^{2}\right)} & \geq\left\|M_{g_{a}^{\prime \prime}}\right\|_{\mathcal{S}_{2}\left(\mathcal{D}, A_{2}^{2}\right)}=\left(\sum_{n}\left\|M_{g_{a}^{\prime \prime}} e_{n}\right\|_{A_{2}^{2}}^{2}\right)^{1 / 2} \\
& =\left(\sum_{n} \int_{\mathbb{D}}\left|g_{a}^{\prime \prime}(z)\right|^{2}\left|e_{n}(z)\right|^{2} d A_{2}(z)\right)^{1 / 2} \\
& =\left(\int_{\mathbb{D}}\left|g_{a}^{\prime \prime}(z)\right|^{2} \log \frac{e}{1-|z|^{2}} d A_{2}(z)\right)^{1 / 2} \\
& \geq C\left(1-|a|^{2}\right)^{-\gamma}\left(\log \frac{e}{1-|a|^{2}}\right)^{1 / 2} .
\end{aligned}
$$

All together yields

$$
\left\|M_{g_{a}^{\prime \prime}}\right\|_{\mathcal{S}_{1}\left(\mathcal{D}, A_{2}^{2}\right)} \asymp\left(1-|a|^{2}\right)^{-\gamma}\left(\log \frac{e}{1-|a|^{2}}\right)^{1 / 2} .
$$

Now, from that and (5.12), we see that the sufficient condition in part (c) is sharp in a certain sense. Also, since $\left\|g_{a}\right\|_{B_{1}} \asymp\left(1-|a|^{2}\right)^{-\gamma}$, we see that part (b) can not be reversed.

To see that part (c) can not be reversed, consider a lacunary series $g(z)=$ $\sum_{k=0}^{\infty} a_{k} z^{n_{k}}$ with $n_{k+1} / n_{k} \geq c>1$. Now, we claim that, if $\sum_{k} n_{k}\left|a_{k}\right|<\infty$ then $M_{g^{\prime \prime}}$ belongs to the trace class $\mathcal{S}_{1}\left(\mathcal{D}, A_{2}^{2}\right)$. Indeed,

$$
\left\|M_{g^{\prime \prime}}\right\|_{\mathcal{S}_{1}}=\left\|\sum_{k=1}^{\infty} n_{k}\left(n_{k}-1\right) a_{k} M_{z^{n} k^{-2}}\right\|_{\mathcal{S}_{1}} \leq C \sum_{k} n_{k}^{2}\left|a_{k}\right|\left\|M_{z^{n} k^{-2}}\right\|_{\mathcal{S}_{1}} .
$$

Since $\left\{e_{n}\right\}_{n \geq 0}=\left\{\frac{z^{n}}{(n+1)^{1 / 2}}\right\}_{n \geq 0}$ is an orthonormal basis of $\mathcal{D}$ and $\left\{\sigma_{n}\right\}_{n \geq 0}=$ $\left\{c_{n} z^{n}\right\}_{n \geq 0}$ with $c_{n} \asymp(n+1)^{3 / 2}$ is an orthonormal basis of $A_{2}^{2}$, an easy computation gives that, for $f(z)=\sum_{n=0}^{\infty} c_{n} z^{n}$, the multiplication operator $M_{z^{j}}$ has the decomposition

$$
M_{z^{j}}(f)=\sum_{n=j}^{\infty} \frac{1}{c_{n}(n-j+1)^{1 / 2}}\left\langle f, e_{n-j}\right\rangle_{\mathcal{D}} \sigma_{n}
$$

and therefore, the singular numbers $\lambda_{n}$ satisfy that $\lambda_{n} \asymp \sqrt{\frac{1}{(n+1)^{3}(n-j+1)}}$. Thus,

$$
\left\|M_{z^{j}}\right\|_{\mathcal{S}_{1}}=\sum_{n \geq j} \lambda_{n} \asymp \sum_{n \geq j} \sqrt{\frac{1}{(n+1)^{3}(n-j+1)}} \asymp j^{-1},
$$


and putting this into (5.13) gives

$$
\left\|M_{g^{\prime \prime}}\right\|_{\mathcal{S}_{1}} \leq C \sum_{k} n_{k}\left|a_{k}\right|
$$

This shows together with part $(b)$ that given a lacunary series $g(z)=$ $\sum_{k} a_{k} z^{n_{k}}$, the multiplication operator $M_{g^{\prime \prime}}: \mathcal{D} \rightarrow A_{2}^{2}$ belongs to $\mathcal{S}_{1}$ if and only if $\sum_{k} n_{k}\left|a_{k}\right|<\infty$, and it is well known that this condition is equivalent to $g$ being in $B_{1}$ [30, p. 100].

Now, given a function $\varphi$ as described in part (d), it is straightforward to select the numbers $\left\{a_{k}\right\}$ and the sequence $\left\{n_{k}\right\}$ so that the summability condition $\sum_{k} n_{k}\left|a_{k}\right|<\infty$ is met, but $\int_{\mathbb{D}}\left|g^{\prime \prime}\right| \varphi d A=\infty$.

Acknowledgments: The authors would like to thank the referee for his/her comments and suggestions that improved the final version of the paper, and also for pointing out the relation between the integration operator $T_{g}$ and the previous work of A. Calderón.

\section{REFERENCES}

[1] J. Arazy, S. Fisher and J. Peetre, Hankel operators on weighted Bergman spaces, Amer. J. Math. 110 (1988), 989-1053.

[2] A. Aleman, A class of integral operators on spaces of analytic functions, Topics in Complex Analysis and Operator Theory, 3-30, Univ. Málaga, Málaga, 2007.

[3] A. Aleman and J. A. Cima, An integral operator on $H^{p}$ and Hardy's inequality, J. Anal. Math. 85 (2001), 157-176.

[4] A. Aleman and A. Siskakis, An integral operator on $H^{p}$, Complex Variables 28 (1995), $149-158$.

[5] A. Aleman and A. Siskakis, Integration operators on Bergman spaces, Indiana Univ. Math. J. 46 (1997), 337-356.

[6] N. Arcozzi, R. Rochberg, E. Sawyer and B. Wick, Function spaces related to the Dirichlet space, J. London Math. Soc. 83 (2011), 1-18.

[7] D. Blasi and J. Pau, A characterization of Besov-type spaces and applications to Hankel-type operators, Michigan Math. J. 56 (2008), 401-417.

[8] A. Calderón, Commutators of singular integral operators, Proc. Nat. Acad. Sci. 53, (1965) 1092-1099.

[9] Z. Harper and M.P. Smith, Testing Schatten class Hankel operators, Carleson embeddings and weighted composition operators on reproducing kernels, J. Operator Theory 55 (2006), 349-371.

[10] D. H. Luecking, Trace ideal criteria for Toeplitz operators, J. Funct. Anal. 73 (1987), 345-368.

[11] D. H. Luecking and K. Zhu, Composition operators belonging to the Schatten ideals, Amer. J. Math. 114 (1992), 1127-1145.

[12] M. Mateljevic and M. Pavlovic, $L^{p}$ - behaviour of power series with positive coeffcients and Hardy spaces, Proc. Amer. Math. Soc. 87 (1983), 309-316.

[13] J. M. Ortega and J. Fàbrega, Pointwise multipliers and corona type decomposition in BMOA, Ann. Inst. Fourier (Grenoble) 46 (1996), 111-137.

[14] J. Pau and J. A. Peláez, Embedding theorems and integration operators on Bergman spaces with rapidly decreasing weights, J. Funct. Anal. 259 (2010), 2727-2756.

[15] M. Pavlović and J. A. Peláez, An equivalence for weighted integrals of an analytic function and its derivative, Math. Nachr. 281 (2008), 1612-1623. 
[16] J. A. Peláez and J. Rättyä, Weighted Bergman spaces induced by rapidly increasing weights, Mem. Amer. Math. Soc., to appear.

[17] C. Pommerenke, Schlichte funktionen und analytische funktionen von beschränkter mittlerer oszillation, Comment. Math. Helv. 52 (1977), 591-602.

[18] S. Pott and M. Smith, Paraproducts and Hankel operators of Schatten class via p-John-Nirenberg Theorem, J. Funct. Anal. 217 (2004), 38-78.

[19] R. Rochberg and Z. Wu, Toeplitz operators on Dirichlet spaces, Int. Equ Oper. Th. 15 (1992), 325-342.

[20] R. Rochberg and Z. Wu, A new characterization of Dirichlet type spaces and applications, Illinois J. Math 37 (1993), 101-122.

[21] B. Simon, Trace Ideals and Their Applications, Cambridge University Press, 1979.

[22] A. Siskakis, Volterra operators on spaces of analytic functions- a survey, Proceedings of the First Advanced Course in Operator Theory and Complex Analysis, 51-68, Univ. Sevilla Secr. Publ., Seville, 2006.

[23] A. Siskakis and R. Zhao, A Volterra type operator on spaces of analytic functions, Function Spaces, Contemp. Math. 232, Amer. Math. Soc., Providence, RI, 1999, 299311.

[24] M.P. Smith, Testing Schatten class Hankel operators and Carleson embeddings via reproducing kernels, J. London Math. Soc. 71 (2005), 172-186.

[25] E. M. Stein, Singular Integrals: The Roles of Calderón and Zygmund. Notices of the AMS 45 n. 9, (1998) 1130-1140.

[26] Z. Wu, Hankel and Toeplitz operators on Dirichlet spaces, Int. Equ Oper. Th. 15 (1992), 503-525.

[27] Z. Wu, Boundedness, compactness and Schatten p-classes of Hankel operators between weighted Dirichlet spaces, Ark. Mat. 31 (1993), 395-417.

[28] R. Zhao and K. Zhu, Theory of Bergman spaces in the unit ball of $\mathbb{C}^{n}$, Mem. Soc. Math. Fr. 115, 2008.

[29] K. Zhu, Analytic Besov spaces, J. Math. Anal. Appl. 157 (1991), 318-336.

[30] K. Zhu, Operator Theory in Function Spaces, Second Edition, Math. Surveys and Monographs, Vol. 138, American Mathematical Society: Providence, Rhode Island, 2007.

Jordi Pau, Departament de Matemàtica Aplicada i Analisi, Universitat de Barcelona, Gran Via 585, 08007 Barcelona, Spain

E-mail address: jordi.pau@ub.edu

José Ángel Peláez, Departamento de Análisis Matemático, Universidad de Málaga, Campus de Teatinos, 29071 Málaga, Spain

E-mail address: japelaez@uma.es 